\title{
Nonstabilized Nielsen coincidence invariants and Hopf-Ganea homomorphisms
}

\author{
ULRICH KOSCHORKE
}

\begin{abstract}
In classical fixed point and coincidence theory the notion of Nielsen numbers has proved to be extremely fruitful. We extend it to pairs $\left(f_{1}, f_{2}\right)$ of maps between manifolds of arbitrary dimensions, using nonstabilized normal bordism theory as our main tool. This leads to estimates of the minimum numbers $\operatorname{MCC}\left(f_{1}, f_{2}\right)$ (and $M C\left(f_{1}, f_{2}\right)$, resp.) of path components (and of points, resp.) in the coincidence sets of those pairs of maps which are homotopic to $\left(f_{1}, f_{2}\right)$. Furthermore, we deduce finiteness conditions for $M C\left(f_{1}, f_{2}\right)$. As an application we compute both minimum numbers explicitly in various concrete geometric sample situations.

The Nielsen decomposition of a coincidence set is induced by the decomposition of a certain path space $E\left(f_{1}, f_{2}\right)$ into path components. Its higher dimensional topology captures further crucial geometric coincidence data. In the setting of homotopy groups the resulting invariants are closely related to certain Hopf-Ganea homomorphisms which turn out to yield finiteness obstructions for $M C$.
\end{abstract}

55M20, 55Q25, 55S35, 57R90; 55N22, 55P35, 55Q40

\section{Introduction}

In this paper we develop a coherent geometric approach to coincidence phenomena in arbitrary codimensions. We prove (and extend considerably) results which were announced in part in [18] and [21].

Consider two continuous maps $f_{1}, f_{2}: M \rightarrow N$ between smooth connected manifolds without boundary, of arbitrary positive dimensions $m$ and $n, M$ being compact.

We would like to measure how small (or simple in some sense) the coincidence locus

$$
C\left(f_{1}, f_{2}\right):=\left\{x \in M \mid f_{1}(x)=f_{2}(x)\right\}
$$

can be made by varying $f_{1}$ and $f_{2}$ within their homotopy classes.

One possible measure is the classical minimum number of coincidence points

$$
M C\left(f_{1}, f_{2}\right):=\min \left\{\# C\left(f_{1}^{\prime}, f_{2}^{\prime}\right) \mid f_{1}^{\prime} \sim f_{1}, f_{2}^{\prime} \sim f_{2}\right\}
$$


(cf Bogaty̌̄-Gonçalves-Zieschang [2, 1.1]). It coincides with the minimum number $\min \left\{\# C\left(f_{1}^{\prime}, f_{2}\right) \mid f_{1}^{\prime} \sim f_{1}\right\}$ where only $f_{1}$ is modified by a homotopy (cf Brooks [3]). In particular, in topological fixed point theory (where $M=N$ and $f_{2}=$ identity) this minimum number is the principal object of study (cf Brown [4, page 9]).

However, in higher codimensions the coincidence locus is generically a manifold of dimension $m-n>0$, and $M C\left(f_{1}, f_{2}\right)$ is often infinite (see eg Examples 1.3, 1.13, 5.2 , and 6.11 below). Thus in many situations it seems more meaningful to study the minimum number of coincidence components

$$
\begin{aligned}
\operatorname{MCC}\left(f_{1}, f_{2}\right): & =\min \left\{\# \pi_{0}\left(C\left(f_{1}^{\prime}, f_{2}^{\prime}\right)\right) \mid f_{1}^{\prime} \sim f_{1}, f_{2}^{\prime} \sim f_{2}\right\} \\
& =\min \left\{\# \pi_{0}\left(C\left(f_{1}^{\prime}, f_{2}\right)\right) \mid f_{1}^{\prime} \sim f_{1}\right\}
\end{aligned}
$$

where $\# \pi_{0}\left(C\left(f_{1}^{\prime}, f_{2}^{\prime}\right)\right)$ denotes the (generically finite) number of path components of the indicated coincidence subspace of $M$ (compare Bogatyı̆-Gonçalves-Zieschang [2, page 47 , line 3]).

Definition 1.1 The pair of maps $\left(f_{1}, f_{2}\right)$ is called loose if $M C\left(f_{1}, f_{2}\right)=0$ (or, equivalently, $\left.\operatorname{MCC}\left(f_{1}, f_{2}\right)=0\right)$, ie if the maps $f_{1}$ and $f_{2}$ can be deformed away from one another.

Question How big are $\operatorname{MCC}\left(f_{1}, f_{2}\right)$ and $M C\left(f_{1}, f_{2}\right)$ ? In particular, when do these invariants vanish, ie when is the pair $\left(f_{1}, f_{2}\right)$ loose?

In order to attack this problem let us study the geometry of generic coincidence submanifolds.

After performing an approximation we may assume that the map $\left(f_{1}, f_{2}\right): M \rightarrow N \times N$ is smooth and transverse to the diagonal $\Delta=\{(y, y) \in N \times N \mid y \in N\}$.

Then the coincidence locus

$$
C=C\left(f_{1}, f_{2}\right)=\left(f_{1}, f_{2}\right)^{-1}(\Delta)=\left\{x \in M \mid f_{1}(x)=f_{2}(x)\right\}
$$

is a closed smooth $(m-n)$-dimensional submanifold of $M$. It comes with two important data. First there is a commuting diagram of maps

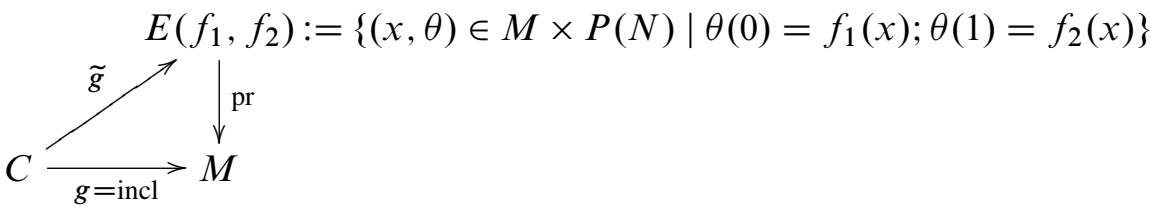


where $P(N)$ (and pr, resp.), denote the space of all continuous paths $\theta:[0,1] \rightarrow N$, endowed with the compact-open topology, (and the obvious projection, resp.); the lifting $\widetilde{g}$ adds the constant path at $f_{1}(x)=f_{2}(x)$ to $g(x)=x \in C$. The second datum is the (composite) vector bundle isomorphism

$$
\bar{g}^{\#}: v(C, M) \cong\left(\left(f_{1}, f_{2}\right) \mid C\right)^{*}(v(\Delta, N \times N)) \cong f_{1}^{*}(T N) \mid C
$$

which describes the normal bundle of $C$ in $M$ (see the figure in Koschorke [21, Section 4] for an illustration).

The resulting bordism class

$$
\omega^{\#}\left(f_{1}, f_{2}\right)=\left[C\left(f_{1}, f_{2}\right), \widetilde{g}, \bar{g}^{\#}\right] \in \Omega^{\#}\left(f_{1}, f_{2}\right)
$$

in an appropriate bordism set ( $\operatorname{cf}(2-1)$ and (2-2) below) is our key coincidence invariant. It turns out that the lifting $\tilde{g}$ plays a crucial role. Indeed, in general the path space $E\left(f_{1}, f_{2}\right)$ (cf (1-5)) has a very rich topology involving both $M$ and the loop space of $N$ (cf Koschorke [22, 2.1]). Already the set $\pi_{0}\left(E\left(f_{1}, f_{2}\right)\right)$ of path components can be huge - it corresponds bijectively to the Reidemeister set

$$
R\left(f_{1}, f_{2}\right)=\pi_{1}(N) / \text { Reidemeister equivalence }
$$

(compare Bogatyı̆-Gonçalves-Zieschang [2, 3.1] and Koschorke [22, 2.1]) which is of central importance in classical Nielsen theory. This leads to a natural decomposition

$$
C\left(f_{1}, f_{2}\right)=\coprod_{A \in \pi_{0}\left(E\left(f_{1}, f_{2}\right)\right)} \tilde{g}^{-1}(A) .
$$

We define $N^{\#}\left(f_{1}, f_{2}\right)$ to be the corresponding number of nontrivial contributions by the various path components $A$ of $E\left(f_{1}, f_{2}\right)$ to $\omega^{\#}\left(f_{1}, f_{2}\right)$ (see Definition 2.2 below).

If we forget the fact that the manifold $C\left(f_{1}, f_{2}\right)$ is embedded in $M$ and if we stabilize $\bar{g}^{\#}$ to yield only a description of the stable normal bundle of $C\left(f_{1}, f_{2}\right)$ we obtain the normal bordism class

(1-9) $\tilde{\omega}\left(f_{1}, f_{2}\right)=\left[C\left(f_{1}, f_{2}\right), \tilde{g}, \bar{g}\right] \in \Omega_{m-n}\left(E\left(f_{1}, f_{2}\right) ; \widetilde{\varphi}:=\operatorname{pr}^{*}\left(f_{1}^{*}(T N)-T M\right)\right)$

and the corresponding Nielsen number $N\left(f_{1}, f_{2}\right)$. These "stabilized" invariants were studied in detail in [22].

Let us put our approach into perspective. Recall the decisive progress made by $\mathbf{J}$ Nielsen on the classical minimizing problem when he decomposed fixed point sets into equivalence classes. In our interpretation this is just the decomposition of a 0 dimensional bordism class according to the path components of its target space. In higher (co)dimensions $(m-n)$ the map $\tilde{g}$ into $E\left(f_{1}, f_{2}\right)$ and the "twisted framing" 
$\bar{g}^{\#}$ contain much more information and lead sometimes to a complete calculation of $\operatorname{MCC}\left(f_{1}, f_{2}\right)$ and $\operatorname{MC}\left(f_{1}, f_{2}\right)$ (cf, for example, Example 1.13 below or Koschorke [21, Examples I-IV]).

Theorem 1.2 (i) The Nielsen numbers $N\left(f_{1}, f_{2}\right)$ and $N^{\#}\left(f_{1}, f_{2}\right)$ are finite and depend only on the homotopy classes of $f_{1}$ and $f_{2}$;

(ii) $N\left(f_{1}, f_{2}\right)=N\left(f_{2}, f_{1}\right)$ and $N^{\#}\left(f_{1}, f_{2}\right)=N^{\#}\left(f_{2}, f_{1}\right)$;

(iii) $0 \leq N\left(f_{1}, f_{2}\right) \leq N^{\#}\left(f_{1}, f_{2}\right) \leq M C C\left(f_{1}, f_{2}\right) \leq M C\left(f_{1}, f_{2}\right)$; if $n \neq 2$, then also

$$
\operatorname{MCC}\left(f_{1}, f_{2}\right) \leq \# \pi_{0}\left(E\left(f_{1}, f_{2}\right)\right)
$$

if $(m, n) \neq(2,2)$, then

$$
M C\left(f_{1}, f_{2}\right) \leq \# \pi_{0}\left(E\left(f_{1}, f_{2}\right)\right) \quad \text { or } \quad M C\left(f_{1}, f_{2}\right)=\infty ;
$$

(iv) if $m=n$, then $N\left(f_{1}, f_{2}\right)=N^{\#}\left(f_{1}, f_{2}\right)$ coincides with the classical Nielsen number (cf Bogatyı̌-Gonçalves-Zieschang [2, Definition 3.6]).

The proof and further details concerning our $\omega$-invariants and Nielsen numbers will be given in Section 2 below. Section 3 is dedicated to the minimum number $\operatorname{MC}\left(f_{1}, f_{2}\right)$.

Example 1.3 Assume $N=S^{1}$. Then both Nielsen numbers of $\left(f_{1}, f_{2}\right)$ agree with $\operatorname{MCC}\left(f_{1}, f_{2}\right)$ and are characterized by the identity

$$
\left(f_{1 *}-f_{2 *}\right)\left(H_{1}(M ; \mathbb{Z})\right)=N^{(\#)}\left(f_{1}, f_{2}\right) \cdot H_{1}\left(S^{1} ; \mathbb{Z}\right) .
$$

If $f_{1} \sim f_{2}$, then $\# \pi_{0}\left(E\left(f_{1}, f_{2}\right)\right)=\infty$ and

$$
N\left(f_{1}, f_{2}\right)=N^{\#}\left(f_{1}, f_{2}\right)=\operatorname{MCC}\left(f_{1}, f_{2}\right)=\operatorname{MC}\left(f_{1}, f_{2}\right)=0 .
$$

If $f_{1}$ and $f_{2}$ are not homotopic then

$$
N\left(f_{1}, f_{2}\right)=N^{\#}\left(f_{1}, f_{2}\right)=\operatorname{MCC}\left(f_{1}, f_{2}\right)=\# \pi_{0}\left(E\left(f_{1}, f_{2}\right)\right) \neq 0
$$

and

$$
\operatorname{MC}\left(f_{1}, f_{2}\right)= \begin{cases}N\left(f_{1}, f_{2}\right) & \text { if } m=1 \\ \infty & \text { if } m \geq 2\end{cases}
$$

(Clearly in all other cases where $m=1$ or $n=1$ we have

$$
\left.N\left(f_{1}, f_{2}\right)=N^{\#}\left(f_{1}, f_{2}\right)=\operatorname{MCC}\left(f_{1}, f_{2}\right)=\operatorname{MC}\left(f_{1}, f_{2}\right)=0 .\right)
$$


In higher codimensional coincidence theory two settings are of particular interest. In one of them, the so-called root case (cf Bogaty̌̆-Gonçalves-Zieschang [2, page 69]) $f_{2}=*$ is constant (henceforth our notation will not distinguish between constant maps and their values). Here our invariants yield the "degrees"

$$
\operatorname{deg}^{\#}(f)=\omega^{\#}(f, *), \quad \widetilde{\operatorname{deg}}(f)=\widetilde{\omega}(f, *)
$$

of a given map $f: M \rightarrow N$. The choice of the constant $* \in N$ is not truly significant since any path joining two such constants induces a bijection between the corresponding bordism sets which is compatible with degrees and Reidemeister decompositions. Note also that $E(f, *)$ is the mapping fiber of $f$ (cf Whitehead [24, I.7])

The second particularly interesting setting concerns selfcoincidences (where $f_{1}$ is equal or at least homotopic to $\left.f_{2}\right)$. Here we know from the very outset that $\operatorname{MCC}\left(f_{1}, f_{2}\right) \leq$ 1 (since $\left.C\left(f_{1}, f_{1}\right)=M\right)$. The remaining question whether $\operatorname{MCC}\left(f_{1}, f_{2}\right)=0$ or, equivalently, whether $f_{1}$ can be deformed away from itself was studied in [20] (and related - in one particular example - to a fascinating problem concerning Lie groups and their role in homotopy theory). It is also worthwhile noting that each of the selfcoincidence invariants $\omega^{\#}(f, f)$ and $\widetilde{\omega}(f, f)$ is determined by the corresponding degree (cf (5-3) and Proposition 5.1 below).

Now let a map $f: M \rightarrow N$ and a constant $* \in N$ be given.

Theorem 1.4 Consider the pairs $\left(f_{1}, f_{2}\right)=(f, *)$ (root case) and $\left(f_{1}, f_{2}\right)=(f, f)$ (selfcoincidence case) simultaneously. Define $b\left(f_{1}, f_{2}\right)$ by $b(f, *):=\# \pi_{0}(E(f, *))$ $=$ index of $f_{*}\left(\pi_{1}(M)\right)$ in $\pi_{1}(N)$, and $b(f, f):=1$.

In both cases the following holds:

(i) If $\omega^{\#}\left(f_{1}, f_{2}\right) \neq 0$ then $N^{\#}\left(f_{1}, f_{2}\right)=b\left(f_{1}, f_{2}\right)$; if in addition $n \neq 2$ then this Nielsen number agrees with $\operatorname{MCC}\left(f_{1}, f_{2}\right)$ (and also with the minimum number $M C\left(f_{1}, f_{2}\right)$ whenever it is finite $)$.

(ii) If even $\widetilde{\omega}\left(f_{1}, f_{2}\right) \neq 0$ then also $N\left(f_{1}, f_{2}\right)=N^{\#}\left(f_{1}, f_{2}\right)$.

In particular in both cases the Nielsen numbers $N^{\#}\left(f_{1}, f_{2}\right)$ and $N\left(f_{1}, f_{2}\right)$ can take only the values 0 and $b\left(f_{1}, f_{2}\right)$.

This has important consequences. First of all since $b(f, *)$ depends on $f$ but $b(f, f)$ does not, the compatibilities with covering spaces must be different in the root and selfcoincidence settings. 
Proposition 1.5 If $\tilde{f}: M \rightarrow \tilde{N}$ is a lifting of $f$ to any connected $d$-fold covering space $\tilde{N}$ of $N$ and $\tilde{*} \in \tilde{N}, * \in N$ are arbitrary constants, then

$$
N^{\#}(f, *)=d \cdot N^{\#}(\tilde{f}, \widetilde{*}) \quad \text { but } N^{\#}(f, f)=N^{\#}(\tilde{f}, \tilde{f})
$$

and the corresponding identities hold also for the weaker (stabilized) Nielsen numbers $N(f, *)$ and $N(f, f)$.

(This holds even when $d=\infty$, provided we define $\infty \cdot 0=0$ ).

Remark 1.6 If $\operatorname{deg}^{\#}(f)$ and $\omega^{\#}(f, f)$, resp., do not vanish and $n \neq 2$, then these identities still hold when we replace $N^{\#}$ by $M C C$ (and by $M C$, provided $M C(f, *)$ and $M C(f, f)$, resp., are finite; compare Theorem 1.4). In general, however, we can only establish the inequalities

$$
M C(f, *) \geq d \cdot M C(\tilde{f}, \tilde{*}) \quad \text { and } \quad M C(f, f) \geq M C(\tilde{f}, \tilde{f}) .
$$

At least in the root case the corresponding equality is often not valid. Indeed there are many examples where $\operatorname{deg}^{\#}(f) \neq 0$ and $M C(\tilde{f}, \widetilde{*})=1$ while $M C(f, *)$ (but not $d$ ) is infinite (see Examples 1.13 and 6.11 below). Such phenomena are closely related to Wyler's theory of injective points (cf Wyler [25] and our discussion following Corollary $6.10)$.

Theorem 1.4, Proposition 1.5, and further results concerning the root and selfcoincidence settings will be proved in Sections 4 and 5 below (cf Remark 4.5 and the discussions following Proposition 4.6 and (5-3)).

As an illustration we test our approach in Section 6 in the case where $M$ is a sphere. Here we can exploit two important advantages. On one hand there is a natural identification of the bordism set $\Omega^{\#}\left(f_{1}, f_{2}\right)$ with a fixed group which does not vary with $f_{1}$ and $f_{2}$. On the other hand the algebraic structure of homotopy groups yields a certain homogeneity; this allows considerable extensions of results which originally are characteristic for the root setting. First we need to recall the following notion.

Definition 1.7 (cf Brown-Schirmer [5]) A map $f: M \rightarrow N$ is not coincidence producing if there exists another map $\bar{f}: M \rightarrow N$ such that the pair $(f, \bar{f})$ is loose (cf Definition 1.1).

Such is always the case when $N$ allows a fixed point free selfmap $a: N \rightarrow N$, eg when the manifold $N$ is open or its Euler number $\chi(N)$ vanishes or $N=S^{n}$ or $N$ is the total space of a nontrivial covering. (On the other hand one can easily exhibit settings where a vast majority of maps is coincidence producing; see eg (6-9) below). 
In the case $M=S^{m}$ non coincidence producing maps determine the subgroup

$$
\pi_{m}^{(2)}(N)=\operatorname{im} p_{*}=\operatorname{ker} \partial \subset \pi_{m}(N)
$$

which arises also naturally in the exact homotopy sequence

$$
\cdots \longrightarrow \pi_{m}\left(\widetilde{C}_{2}(N)\right) \stackrel{p_{*}}{\longrightarrow} \pi_{m}(N) \stackrel{\partial}{\longrightarrow} \pi_{m-1}(N-\{*\}) \longrightarrow \cdots
$$

of the fibration $p: \widetilde{C}_{2}(N) \rightarrow N$ of the configuration space of ordered pairs of distinct points in $N$.

Theorem 1.8 Given $m \geq 1$, consider maps $f_{i}: S^{m} \rightarrow N, i=1,2$, which are not both coincidence producing. Assume $\omega^{\#}\left(f_{1}, f_{2}\right) \neq 0$.

Then

$$
N^{\#}\left(f_{1}, f_{2}\right)=\# \pi_{0}\left(E\left(f_{1}, f_{2}\right)\right)=\left[\pi_{1}(N):\left(f_{1 *}-f_{2 *}\right)\left(\pi_{1}\left(S^{m}\right)\right)\right] .
$$

If in addition $n \neq 2$, then this Nielsen number agrees with $\operatorname{MCC}\left(f_{1}, f_{2}\right)$ (and also with the minimum number $M C\left(f_{1}, f_{2}\right)$ whenever it is finite).

If $\widetilde{\omega}\left(f_{1}, f_{2}\right) \neq 0$ and $n \geq 1$, then also $N\left(f_{1}, f_{2}\right)=N^{\#}\left(f, f_{2}\right)$.

Corollary 1.9 If $f: S^{m} \rightarrow N$ is not coincidence producing and $\pi_{1}(N) \neq 0$ then $\omega^{\#}(f, f)=0$.

Indeed otherwise $N^{\#}(f, f)$ would have to agree both with $\# \pi_{1}(N)$ and with 1 . This highlights a rather astonishing feature of our invariant in the non simply connected case: if $f$ can be deformed away from any map, $\omega^{\#}$ behaves as if $f$ can be deformed away from itself.

Corollary 1.10 If $\pi_{1}(N)$ has a nontrivial proper subgroup $G$ then $\omega^{\#}(f, f)=0$ for every map $f: S^{m} \rightarrow N$.

Indeed $G$ corresponds to a nontrivial covering space $\tilde{N}$ of $N$ with $\pi_{1}(\tilde{N}) \neq 0$. Thus a lifting $\tilde{f}$ of $f$ is not coincidence producing and we have here $0=N^{\#}(\tilde{f}, \tilde{f})=$ $N^{\#}(f, f)$ (cf Proposition 1.5).

For certain specific target manifolds $N$ (eg when $N$ is open or when $N \neq S^{1}$ has an infinite fundamental group or when $N$ is a nontrivial product of manifolds) it is rather easy to see that all pairs of maps $f_{1}, f_{2}: S^{m} \rightarrow N$ are loose (see Corollary 6.3 below). In view of Theorem 1.8 a more systematic and detailed discussion is desirable: what happens in general when $\omega^{\#}\left(f_{1}, f_{2}\right)$ vanishes? It is reasonable to concentrate 
first on the root case. Given $m \geq 1$, we define (in Definition 6.4) an abelian group $X_{m}(N)$ which measures to some extend the comparative strength of the Nielsen number $N^{\#}(f, *)$ on one hand and of $\operatorname{MCC}(f, *)$ on the other hand, taking into account all maps $f: S^{m} \rightarrow N$. This group vanishes precisely if the conditions $N^{\#}(f, *)=0$ and $\operatorname{MCC}(f, *)=0$ are equivalent. Actually this is often satisfied (cf Theorem 6.5 below).

Theorem 1.11 Given $m \geq 1$, assume $X_{m}(N)=0$. Then a pair of maps $f_{1}, f_{2}: S^{m} \rightarrow$ $N$ is loose if and only if $\omega^{\#}\left(f_{1}, f_{2}\right)=0$ and at least one of the maps $f_{1}, f_{2}$ is not coincidence producing.

Thus, if in the situation of Theorem $1.8 X_{m}(N)$ and $\omega^{\#}\left(f_{1}, f_{2}\right)$ vanishes, then so do also $N^{\#}\left(f_{1}, f_{2}\right), \operatorname{MCC}\left(f_{1}, f_{2}\right)$ and $\operatorname{MC}\left(f_{1}, f_{2}\right)$.

Example 1.12 $\left(\left(N=S^{n}\right)\right)$ Consider maps $f_{1}, f_{2}: S^{m} \rightarrow S^{n}$ where $m, n \geq 1$, and let $a$ denote the antipodal involution. Then

$$
\operatorname{MCC}\left(f_{1}, f_{2}\right)=N^{\#}\left(f_{1}, f_{2}\right)= \begin{cases}0 & \text { if } f_{i} \sim a f_{2} \\ \# \pi_{0}\left(E\left(f_{1}, f_{2}\right)\right) & \text { otherwise }\end{cases}
$$

If $f_{1} \not \subset a f_{2}$ then $\# \pi_{0}\left(E\left(f_{1}, f_{2}\right)\right)$ equals 1 (and $\left|d^{0}\left(f_{1}\right)-d^{0}\left(f_{2}\right)\right|$, resp.) according as $n \neq 1$ (or $m=n=1$, resp.; here $d^{0}\left(f_{i}\right) \in \mathbb{Z}$ is the usual degree).

This shows that the "strong" Nielsen number $N^{\#}\left(f_{1}, f_{2}\right)$ (based on the nonstabilized invariant $\left.\omega^{\#}\left(f_{1}, f_{2}\right)\right)$ is often strictly larger than $N\left(f_{1}, f_{2}\right)$. For example, [22, Corollary 1.17] contains a long list of dimension combinations $(m, n)$ such there exists a map $f: S^{m} \rightarrow S^{n}$ with $N(f, *)=0$ but $\operatorname{MCC}(f, *)=1$.

Clearly $\operatorname{MC}\left(f_{1}, f_{2}\right) \leq 1$ whenever $\left[f_{1}\right]-\left[a \circ f_{2}\right]$ lies in $E\left(\pi_{m-1}\left(S^{n-1}\right)\right)$, the image of the Freudenthal suspension. On the other hand, it is well known that $M C\left(f_{1}, f_{2}\right)$ is infinite if $\left[f_{1}\right]-\left[a \circ f_{2}\right] \notin E\left(\pi_{m-1}\left(S^{n-1}\right)\right)$ and $m, n \neq(1,1)$. This follows also as a very special consequence of one of our results concerning $M C$ (cf Theorem 3.1(i)).

In Section 3 we discuss in great generality lower and upper bounds for $M C\left(f_{1}, f_{2}\right)$ (and deduce the classical Wecken theorem for coincidences as a corollary). In particular, in the case $M=S^{m}$ we obtain a necessary (and, if $X_{m}(N)=0$, also sufficient) finiteness condition for $M C\left(f_{1}, f_{2}\right)$, expressed in terms of $\omega^{\#}\left(f_{1}, f_{2}\right)$ (cf Theorem 6.9 below). 
Example 1.13 Let $N$ be an odd-dimensional spherical space form (ie the quotient of $S^{n}$ by a free action of a finite group $G$ ). Then we have for all $f_{1}, f_{2}: S^{m} \rightarrow N$ :

$$
\operatorname{MCC}\left(f_{1}, f_{2}\right)=N^{\#}\left(f_{1}, f_{2}\right)= \begin{cases}0 & \text { if } f_{1} \sim f_{2} \text { or } m<n ; \\ \# G & \text { if } f_{1} \nsucc f_{2} \text { and } m>1 \\ \left|d^{0}\left(f_{1}\right)-d^{0}\left(f_{2}\right)\right| & \text { if } m=1 \text { and } N=S^{1}\end{cases}
$$

(Here $d^{0}\left(f_{i}\right) \in \mathbb{Z}$ denotes the usual degree).

Moreover, if $n \geq 3$ then

$$
\operatorname{MC}\left(f_{1}, f_{2}\right)= \begin{cases}\infty & \text { if }\left[f_{1}\right]-\left[f_{2}\right] \notin p_{*} \circ E(\pi) \\ 0 & \text { if } f_{1} \sim f_{2} \text { or } m<n \\ \# G & \text { otherwise. }\end{cases}
$$

Here

$$
\pi \subset \pi_{m-1}\left(S^{n-1}\right) \stackrel{E}{\longrightarrow} \pi_{m}\left(S^{n}\right) \stackrel{p_{*}}{\longrightarrow} \pi_{m}(N)
$$

are the natural (eg suspension) homomorphisms and $\pi$ denotes all of $\pi_{m-1}\left(S^{n-1}\right)$ if $\# G \leq 2$, and the kernel of the (total) Hopf-Hilton homomorphism $h$ (cf Corollary 6.10 below) if $\# G \geq 3$.

Observe that no specific feature of the group action - apart from the order of $G$ - enters the picture here. For a geometric explanation of such phenomena in terms of almost injective points see the discussion of Definition 6.12 below.

For further concrete geometric settings where the minimum numbers $M C\left(f_{1}, f_{2}\right)$ and $\operatorname{MCC}\left(f_{1}, f_{2}\right)$ have been calculated explicitly, see Koschorke [21, Examples I-III].

Having the precise finiteness criterion Theorem 6.9 for $M C\left(f_{1}, f_{2}\right)$ at our disposal we may ask: what can we say in case it is satisfied?

Theorem 1.14 Consider maps $f_{1}, f_{2}: S^{m} \rightarrow N$ into an arbitrary $n$-manifold such that $(m, n) \neq(2,2)$. Assume that $M C\left(f_{1}, f_{2}\right)$ is finite. If the suspension

$$
E: \pi_{m-1}\left(S^{n-1}\right) \rightarrow \pi_{m}\left(S^{n}\right)
$$

is injective (eg if $m<2 n-2$ or $n=2$ ), then

$$
N^{\#}\left(f_{1}, f_{2}\right)=\operatorname{MCC}\left(f_{1}, f_{2}\right)=\operatorname{MC}\left(f_{1}, f_{2}\right) \leq \# \pi_{1}(N) .
$$

This follows from Theorem 6.14 below.

At the end of this paper we give purely homotopy theoretical descriptions of our basic geometric coincidence invariant deg" ${ }^{\#}$ (cf Theorem 7.2 and Corollary 7.3). We 
obtain a decomposition of $\mathrm{deg}^{\#}$ into two components ( $\mathrm{cf}(7-8)$ ). One of them is the Ganea-Hopf invariant $H_{\mathcal{C}}$ relative to an attaching map of top dimensional cells in the universal covering space $\tilde{N}$ of $N$. It turns out that $H_{\mathcal{C}}$ is a finiteness obstruction for the minimum number $M C$ (and actually the only one in a dimension range depending on the connectivity of $N$, cf Corollary 7.4 and Theorem 7.6). Here we use homotopy theoretical tools such as Ganea's exact EHP-sequence.

In much of our discussions we can switch freely back and forth between base point preserving maps and homotopies and their base point free counterparts. This is made precise in Appendix A.

The approach of this paper can also be applied fruitfully to general inverse image problems (where the submanifolds $\{*\} \subset N$ and $\Delta \subset N \times N$, cf (1-4), are replaced by arbitrary closed smooth submanifolds) or to over- and under-crossings of link maps into a manifold of the form $N \times \mathbb{R}$. In the latter case we obtain unlinking obstructions which often settle unlinking questions and which, in addition, turn out to distinguish a great number of different link homotopy classes (and sometimes even classify them completely). Moreover, our approach also leads to the notion of Nielsen numbers for link maps (cf Koschorke [19]).

Conventions 1.15 All manifolds are assumed to be Hausdorff spaces having a countable basis; they have empty boundaries unless stated otherwise. A submanifold $C \subset M$ with a specified trivialization of its normal bundle $v(C, M)$ is called framed. In any bordism set 0 denotes the class represented by empty data. We will often neglect the notational distinction between constant maps and their values. Given any topological space $X, P(X)$ is the space of all paths $\theta: I \rightarrow X$, endowed with the compact-open topology. \#S denotes the (finite or infinite) number of elements in a set $S . E$ stands for Freudenthal suspension. $\varphi:=f_{1}^{*}(T N)-T M \in K O(M)$ and $\tilde{\varphi}:=\operatorname{pr}^{*}(\varphi) \in K O\left(E\left(f_{1}, f_{2}\right)\right)$ (compare (1-9)) are the relevant virtual coefficient bundles for our (stabilized) obstruction theory. Arbitrary reflections on spheres are denoted by $r$.

\section{The strong $\omega$-invariant $\omega^{\#}\left(f_{1}, f_{2}\right)$ and the strong Nielsen number $N^{\#}\left(f_{1}, f_{2}\right)$}

Throughout this paper $f_{1}, f_{2}, f: M \rightarrow N$ denote (continuous) maps between the smooth connected (non-empty) manifolds $M$ and $N$ without boundary, of strictly positive dimensions $m$ and $n$, resp., $M$ being compact. 
Consider the set

$$
\Omega^{\#}\left(f_{1}, f_{2}\right):=\left\{\left(C, \tilde{g}, \bar{g}^{\#}\right)\right\} / \text { bordism in } M \times I
$$

of bordism classes of triples of the indicated form where

(i) $C$ is a closed smooth submanifold of $M$;

(ii) $\tilde{g}: C \rightarrow E\left(f_{1}, f_{2}\right)$ is a section of $\operatorname{pr} \mid(\operatorname{cf}(1-5))$, ie $\operatorname{pr} \circ \tilde{g}$ is the inclusion;

(iii) $\bar{g}^{\#}: v(C, M) \cong f_{1}^{*}(T N) \mid C$ is a vector bundle isomorphism which gives a (nonstabilized) description of the normal bundle of $C$ in $M$ in terms of the tangent bundle $T N$ of $N$.

Such triples occur very naturally when we study the coincidence behavior of $f_{1}$ and $f_{2}$. Indeed, if the map $\left(f_{1}, f_{2}\right): M \longrightarrow N \times N$ is smooth and transverse to the diagonal $\Delta$ then the coincidence data (1-4)-(1-6) yield the desired triple.

If $f_{1}$ and $f_{2}$ are arbitrary continuous maps, we apply this procedure to a smooth map $\left(f_{1}^{\prime}, f_{2}^{\prime}\right)$ which approximates $\left(f_{1}, f_{2}\right)$ and is transverse to $\Delta$. Using the techniques of [22, Section 3], we see that there is a canonical bijection $\Omega^{\#}\left(f_{1}^{\prime}, f_{2}^{\prime}\right) \approx \Omega^{\#}\left(f_{1}, f_{2}\right)$ induced by any sufficiently small homotopy from $\left(f_{1}^{\prime}, f_{2}^{\prime}\right)$ to $\left(f_{1}, f_{2}\right)$.

In any case the resulting triple $\left(C, \widetilde{g}, \bar{g}^{\#}\right)$ determines a well-defined bordism class

$$
\omega^{\#}\left(f_{1}, f_{2}\right)=\left[C, \widetilde{g}, \bar{g}^{\#}\right] \in \Omega^{\#}\left(f_{1}, f_{2}\right) .
$$

The same kind of argument allows us to handle also arbitrary (possibly "large") homotopies. The result can be best expressed in the language of functors. Consider the category $\mathfrak{P}$ whose objects are continuous maps $\left(f_{1}, f_{2}\right): M \rightarrow N \times N$ and whose morphisms are equivalence classes of homotopies $F:\left(f_{1}, f_{2}\right) \sim\left(f_{1}^{\prime}, f_{2}^{\prime}\right)$; here two homotopies $f_{0}, f_{1}$ from $\left(f_{1}, f_{2}\right)$ to $\left(f_{1}^{\prime}, f_{2}^{\prime}\right)$ are called equivalent if they can be deformed continuously into one another through such homotopies (ie at each stage of the deformation $F_{t}, t \in[0,1]$, is a homotopy from $\left(f_{1}, f_{2}\right)$ to $\left.\left(f_{1}^{\prime}, f_{2}^{\prime}\right)\right)$.

Proposition 2.1 The nonstabilized coincidence invariant determines a functor $\left(\Omega^{\#}, \omega^{\#}\right)$ from the category $\mathfrak{P}$ of pairs of maps and (deformation classes of) homotopies to the category consisting of pointed sets and of bijections preserving the preferred element.

If we consider the coincidence submanifold $C=C\left(f_{1}, f_{2}\right)$ of $M$ just as an abstract manifold and if we stabilize $\bar{g}^{\#}$ to yield the stable vector bundle isomorphism

$$
\bar{g}: T C \oplus f_{1}^{*}(T N)|C \cong T M| C
$$


we obtain the coincidence invariant

$$
\widetilde{\omega}\left(f_{1}, f_{2}\right)=[C, \widetilde{g}, \bar{g}] \in \Omega_{m-n}\left(E\left(f_{1}, f_{2}\right) ; \quad \tilde{\varphi}:=\operatorname{pr}^{*}\left(f_{1}^{*}(T N)-T M\right)\right)
$$

which was studied in [22]. Clearly it, too, determines a functor as above which actually takes values in (normal bordism) groups with a preferred element. Stabilization yields a forgetful transformation

$$
\text { stab: }\left(\Omega^{\#}\left(f_{1}, f_{2}\right), \omega^{\#}\left(f_{1}, f_{2}\right)\right) \longrightarrow\left(\Omega_{m-n}\left(E\left(f_{1}, f_{2}\right) ; \widetilde{\varphi}\right), \widetilde{\omega}\left(f_{1}, f_{2}\right)\right)
$$

In the stable dimension range $m \leq 2 n-2$ we are dealing with bijections here and stabilization leads to no loss of information. (Actually, $\widetilde{\omega}\left(f_{1}, f_{2}\right)$ is even the only looseness obstruction if $m<2 n-2$; $\operatorname{cf}$ [22, Theorem 1.10]). However, in general there are many situations where the nonstabilized coincidence invariant $\omega^{\#}\left(f_{1}, f_{2}\right)$ turns out to be considerably more powerful than $\widetilde{\omega}\left(f_{1}, f_{2}\right)$. (This is reflected by the discussion in Example 1.12). On the other hand it is often much easier to handle the stabilized invariant $\widetilde{\omega}\left(f_{1}, f_{2}\right)$ : it lies in a bordism group (not just set) and computational techniques are available (especially for low codimensions $(m-n)$, cf [17, 9.3]; compare also [21, Section 3]).

Next, given any bordism class $c=\left[C, \widetilde{g}, \bar{g}^{\#}\right] \in \Omega^{\#}\left(f_{1}, f_{2}\right)(\operatorname{cf}(2-1))$, let

$$
c_{A}=\left[C_{A}=\tilde{g}^{-1}(A), \tilde{g}\left|C_{A}, \bar{g}^{\#}\right|\right]
$$

denote its contribution to a given path component $A \in \pi_{0}\left(E\left(f_{1}, f_{2}\right)\right)$.

Definition 2.2 (i) We call a path component $A$ of $E\left(f_{1}, f_{2}\right)$ strongly essential if the corresponding contribution $\omega_{A}^{\#}\left(f_{1}, f_{2}\right)$ to $\omega^{\#}\left(f_{1}, f_{2}\right)$ is nontrivial (ie not representable by empty data).

(ii) We define the strong Nielsen number $N^{\#}\left(f_{1}, f_{2}\right)$ of $f_{1}$ and $f_{2}$ to be the number of strongly essential path components $A \in \pi_{0}\left(E\left(f_{1}, f_{2}\right)\right)$.

This is in analogy to the ("weak") Nielsen number $N\left(f_{1}, f_{2}\right)$ which was extracted from

$$
\widetilde{\omega}\left(f_{1}, f_{2}\right) \in \Omega_{m-n}\left(E\left(f_{1}, f_{2}\right) ; \tilde{\varphi}\right) \cong \bigoplus_{A \in \pi_{0}\left(E\left(f_{1}, f_{2}\right)\right)} \Omega_{m-n}(A ; \tilde{\varphi} \mid A)
$$

and discussed in detail in [22].

Remark 2.3 Clearly, if $\omega^{\#}\left(f_{1}, f_{2}\right)$ is trivial then so is $N^{\#}\left(f_{1}, f_{2}\right)$. However it is conceivable that the converse does not hold in general; indeed, the various components $C_{A}\left(f_{1}, f_{2}\right)$ of the coincidence locus may possibly link in $M$ so that their nulbordisms cannot be fitted together to yield disjoint embeddings into $M \times I$. 
Such complications cannot arise in the stabilized theory: $N\left(f_{1}, f_{2}\right)=0$ if and only if $\widetilde{\omega}\left(f_{1}, f_{2}\right)=0$ (much like a norm in a vector space decides precisely whether a given vector vanishes).

Remark 2.4 In higher codimensions our bordism approach allows us to capture coincidence phenomena which seem to be entirely outside the reach of the methods of singular (co)homology theory. Already the weakened stabilized bordism invariant

$$
\omega\left(f_{1}, f_{2}\right):=\operatorname{pr}_{*}\left(\widetilde{\omega}\left(f_{1}, f_{2}\right)\right) \in \Omega_{m-n}\left(M ; \varphi:=f_{1}^{*}(T N)-T M\right)
$$

(which involves neither the path space $E\left(f_{1}, f_{2}\right)$, cf (1-5), nor the resulting Nielsen decomposition) has lead to the solution of a problem which corresponds to determining cohomological obstructions of arbitrarily high order (cf the theorem in the introduction of [20] and its corollaries).

Theorem 1.2 and Example 1.3 of the introduction follow now from (1-8), (1-10), (2-1), (4-5), (5-2) and from [22, Example I], or they can be proved by refining the methods of that paper (cf also Theorem 3.1(iii) below).

In general the nonnegative integer $N^{\#}\left(f_{1}, f_{2}\right)$ contains considerably less information than the invariant $\omega^{\#}\left(f_{1}, f_{2}\right)$ which, however, has the drawback that it lies in a bordism set which varies with $f_{1}$ and $f_{2}$.

This complication can be avoided in some important settings. Given $y_{0} \in N$, let $\Omega\left(N, y_{0}\right)^{+}$denote the loop space of $N$ at $y_{0}$, with an extra point + added; thus $S^{n} \wedge\left(\Omega\left(N, y_{0}\right)^{+}\right)$is the Thom space of the trivial $n$-plane bundle over $\Omega\left(N, y_{0}\right)$.

Proposition 2.5 (cf Hatcher-Quinn $[12,3.1])$ Assume that $M$ is $(m-n+1)-$ connected. Then any choice of points $x_{0} \in M, y_{0} \in N$ and of paths $\gamma_{i}$ in $N$ joining $f_{i}\left(x_{0}\right)$ to $y_{0}, i=1,2$, and of a local orientation of $N$ at $y_{0}$ induces a bijection

$$
\Omega^{\#}\left(f_{1}, f_{2}\right) \approx\left[M, S^{n} \wedge\left(\Omega\left(N, y_{0}\right)^{+}\right)\right]
$$

(involving the Pontryagin-Thom procedure) and thus allows us to identify the coincidence invariant $\omega^{\#}\left(f_{1}, f_{2}\right)$ with an element of the indicated homotopy set. These identifications commute with the bijections induced by homotopies of $\left(f_{1}, f_{2}\right)$, cf Proposition 2.1, provided the paths in $N$ are chosen compatibly.

Moreover there is a canonical involution inv of the homotopy set $\left[M, S^{n} \wedge\left(\Omega\left(N, y_{0}\right)^{+}\right)\right]$ such that

$$
\omega^{\#}\left(f_{2}, f_{1}\right)=\operatorname{inv}\left(\omega^{\#}\left(f_{1}, f_{2}\right)\right)
$$


Proof Given a triple $\left(C, \widetilde{g}, \bar{g}^{\#}\right)$ as in $(2-1)$, a cell-by-cell argument allows us to construct a homotopy $G: C \times I \rightarrow M$ from the inclusion $g=\operatorname{pr} \circ \tilde{g}: C \subset M$ to the constant map $g_{1}$ at $x_{0}$. A lift of $G$ in $E\left(f_{1}, f_{2}\right)$, starting with $\widetilde{g}$, ends with a map

$$
\tilde{g}_{1}: C \longrightarrow \operatorname{pr}^{-1}\left(\left\{x_{0}\right\}\right) \sim \Omega\left(N, y_{0}\right) ;
$$

(compare diagram (1-5)); if $x \in C$ and $\widetilde{g}(x)=(x, \theta)$ then $\widetilde{g}_{1}(x)$ can be given by the concatenated path

$$
y_{0} \stackrel{\gamma_{1}^{-1}}{\longrightarrow} f_{1}\left(x_{0}\right) \stackrel{f_{1} \circ G(x,-)^{-1}}{\longrightarrow} f_{1}(x) \stackrel{\theta}{\longrightarrow} f_{2}(x) \stackrel{f_{2} \circ G(x,-)}{\longrightarrow} f_{2}\left(x_{0}\right) \stackrel{\gamma_{2}}{\longrightarrow} y_{0} .
$$

$G$ induces also a vector bundle isomorphism (cf $[22,3.1])$.

$$
f_{1}^{*}(T N) \mid C \cong g_{1}^{*}\left(f_{1}^{*}(T N)\right) \cong C \times \mathbb{R}^{n}
$$

which we compose with $\bar{g}^{\#}$ (cf $(2-1)$ ) to obtain a normal framing $\bar{g}_{1}^{\#}$ of $C$ in $M$. The Pontryagin-Thom construction transforms the resulting triple $\left(C, \widetilde{g}_{1}, \bar{g}_{1}^{\#}\right)$ into an element of the homotopy set $\left[M, S^{n} \wedge\left(\Omega\left(N, y_{0}\right)^{+}\right)\right]$.

Our connectivity assumption guarantees that this procedure yields a well defined bijection. It implies also that $n \geq 2$ (since $H_{m}\left(M ; \mathbb{Z}_{2}\right) \neq 0$ ). In particular, the Thom space $S^{n} \wedge\left(\Omega\left(N, y_{0}\right)^{+}\right)$is simply connected and we may identify the elements of $\Omega^{\#}\left(f_{1}, f_{2}\right)$ with base point preserving or base point free homotopy classes, as we wish. (Note also that the choices of $x_{0}, \gamma_{1}$ and $\gamma_{2}$ do not matter in case $N$ is 1-connected).

Next we describe the involution inv of the homotopy set $\left[M, S^{n} \wedge\left(\Omega\left(N, y_{0}\right)^{+}\right)\right]$ at the level of bordism classes. Given a submanifold $C$ of $M$, a map $\widetilde{g}_{1}: C \rightarrow$ $\Omega\left(N, y_{0}\right)$ and a framing $\bar{g}^{\#}: v(C, M) \cong C \times \mathbb{R}^{n}$, evaluate $\widetilde{g}_{1}$ to obtain a homotopy $h: C \times I \rightarrow N$ from the constant map at $y_{0}$ to itself. Choose a trivialization $\bar{h}^{\#}: h^{*}(T N) \stackrel{\cong}{\longrightarrow}(C \times I) \times T_{y_{0}}(N)$ which restricts to the identity over $C \times\{0\}$ and let $\bar{h}_{1}^{\#}$ denote the corresponding automorphism of $C \times T_{y_{0}}(N) \cong C \times \mathbb{R}^{n}$ over $C \times\{1\}=C$. Compose $\bar{g}_{1}^{\#}$ with $-\bar{h}_{1}^{\#}$ and $\tilde{g}_{1}$ with the involution of $\Omega\left(N, y_{0}\right)$ which reverses the loops. The resulting triple represents $\operatorname{inv}\left(\left[C, \widetilde{g}_{1}, \bar{g}_{1}^{\#}\right]\right)$.

Now apply the whole preceding discussion to the special case where $\left(C, \tilde{g}, \bar{g}^{\#}\right)$ are the coincidence data of the (generic) pair $\left(f_{1}, f_{2}\right)$. Interchanging $f_{1}$ and $f_{2}$ clearly reverses the path $\widetilde{g}_{1}(x), x \in C$ (cf (2-9) where $\theta=$ const in this case). Also if we describe $v(C, M)$ by $f_{2}^{*}(T N)$ (instead of $f_{1}^{*}(T N)$, cf (2-1)(iii)) we must base the analogue of (2-10) on a trivialization $\left(f_{2} \circ G\right)^{*}(T N) \cong C \times I \times T_{y_{0}}(N)$. Moreover note that the two projections from the diagonal $\Delta \subset N \times N$ to $N$ yield isomorphisms $v(\Delta, N \times N) \cong T N$ which play a role in (1-6) but differ by a factor -1 . Indeed, for all $y \in N$ and $v \in T_{y}(N)$ the two vectors $(v, 0),(0,-v) \in T_{(y, y)}(N \times N)$ yield the 
same element in $v(\Delta, N \times N)$ since their difference is tangential to $\Delta$. This explains the negative sign in the definition of the involution inv.

Our chosen local orientation of $N$ at the point $y_{0} \in N$ (cf Proposition 2.5) determines a collapsing map

$$
\text { coll: } N \longrightarrow N /\left(N-\stackrel{\circ}{B}^{n}\right) \cong B^{n} / \partial B^{n} \cong S^{n}
$$

up to homotopy (where $B^{n}$ is a small ball around $y_{0}$ ). Consider also the maps

$$
S^{n} \stackrel{\mathrm{in}_{\ell}}{\longrightarrow} S^{n} \wedge\left(\Omega\left(N, y_{0}\right)^{+}\right) \stackrel{\mathrm{ret}^{\prime}}{\longrightarrow} S^{n}
$$

defined by the inclusion at a fixed loop $\ell \in \Omega\left(N, y_{0}\right)$ and by the "horizontal projection". We obtain the diagram of (induced) maps

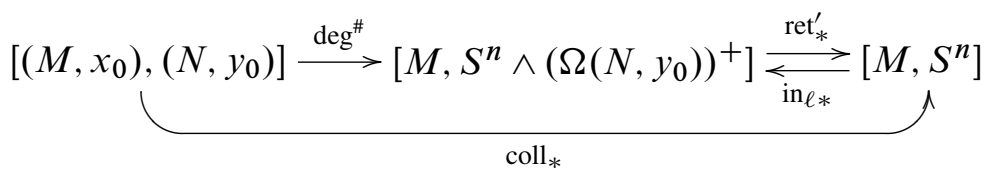

Proposition 2.6 Assume that $M$ is $(m-n+1)$-connected.

Then we have in diagram (2-12)

$$
\begin{aligned}
\operatorname{coll}_{*} & =u_{\varepsilon *} \circ \operatorname{ret}_{*}^{\prime} \circ \text { inv } \circ \operatorname{deg}^{\#} \quad \text { and } \\
\operatorname{ret}_{*}^{\prime} \circ \text { in }_{\ell *} & =\text { identity }
\end{aligned}
$$

where $u_{\varepsilon}$ denotes a selfmap of $S^{n}$ of degree $\varepsilon=(-1)^{n}$ (cf also Proposition 2.5).

Hence for every map $f:\left(M, x_{0}\right) \rightarrow\left(N, y_{0}\right)$ the degree $\operatorname{deg}^{\#}(f)=\omega^{\#}\left(f, y_{0}\right)$ determines the homotopy class of coll $\circ f: M \rightarrow S^{n}$. Moreover the selfcoincidence invariant $\omega^{\#}(f, f)$ lies in the image of $\operatorname{in}_{\ell *}$ for some $\ell \in \Omega\left(N, y_{0}\right)$ and therefore is determined by the seemingly weaker invariant $\operatorname{ret}_{*}^{\prime}\left(\omega^{\#}(f, f)\right)$.

In particular, if $N=S^{n}$ then $\operatorname{deg}^{\#}(f)$ determines $f$ up to homotopy and we have

$$
N^{\#}\left(f, y_{0}\right)=\operatorname{MCC}\left(f, y_{0}\right)=\left\{\begin{array}{ll}
0 & \text { if } f \sim y_{0} \\
1 & \text { if } f \not y_{0}
\end{array} .\right.
$$

Proof Generically $f$ is smooth with regular value $y_{0}$. Represent $\omega^{\#}\left(y_{0}, f\right)=$ $\operatorname{inv}\left(\operatorname{deg}^{\#}(f)\right)(\operatorname{cf}(2-7))$ by the triple $\left(C=C\left(y_{0}, f\right)=f^{-1}\left(\left\{y_{0}\right\}\right), \widetilde{g}_{1}, \bar{g}_{1}^{\#}\right)(\operatorname{cf}(2-8)$, (2-10)) and forget the map $\tilde{g}_{1}: C \rightarrow \Omega\left(N, y_{0}\right)$ (or, equivalently, apply the "horizontal" projection $\left.S^{n} \wedge\left(\Omega\left(N, y_{0}\right)^{+}\right) \rightarrow S^{n}\right)$. Then the data $\left(C,-\bar{g}_{1}^{\#}\right)$ give the standard characterization (in the sense of Pontryagin-Thom) of coll $\circ f$. (For the negative sign compare the proof of Proposition 2.5.) 
An inspection of (2-9) shows that $\tilde{g}_{1}$ is homotopy trivial when $f_{1}=f_{2}$ and $\theta$ is a constant path. Thus $\omega^{\#}(f, f)$ lies in the image of $\operatorname{in}_{\ell *}$ where $\ell=\gamma_{1}^{-1} \gamma_{2}$ (compare Proposition 2.5).

If $N=S^{n}$, then $\# \pi_{0}(E(f, *))=1$ must agree with $N^{\#}(f, *)$ whenever $f \not \psi$ and hence $\operatorname{deg}^{\#}(f)$ is nontrivial. The last claim follows from Theorem 1.2(iii) and can be established also for $n=2$ by a connected sum argument.

\section{Isolated coincidence points}

In this section we establish a finiteness criterion as well as upper bounds for $\operatorname{MC}\left(f_{1}, f_{2}\right)$. In the special case $m=n>2$ we reobtain the classical Wecken theorem.

In view of Example 1.3 we may (and do) assume that $m \geq 2$.

First we construct a map

$$
e: \bigoplus_{A \in \pi_{0}\left(E\left(f_{1}, f_{2}\right)\right)} \pi_{m-1}\left(S^{n-1}\right) \longrightarrow \Omega^{\#}\left(f_{1}, f_{2}\right)
$$

Step I Given a path component $A$ of $E\left(f_{1}, f_{2}\right)$, choose an element $(x, \theta) \in A$ as well as the following data:

(i) a diffeomorphism $b:\left(B^{m}, 0\right) \rightarrow\left(B_{x}, x\right)$ from the standard compact unit ball $B^{m}$ in $\mathbb{R}^{m}$ onto a small "ball" $B_{x}$ around $x$ in $M$; and

(ii) a trivialization $\bar{b}$ of $f_{1}^{*}(T N) \mid B_{x}$, the pullback of the tangent bundle of $N$ by $f_{1} \mid B_{x}$.

These data, together with the inclusion $B_{x} \subset M$, allow us to interpret any framed closed smooth submanifold $C$ of $\stackrel{\circ}{B}^{m}$ as a triple $\left(C, g, \bar{g}^{\#}\right)$ (compare (1-5), (1-6), and (2-1)). Moreover the embedding $g$ : $C \subset M$ lifts canonically (up to homotopy) to a map $\tilde{g}$ into $E\left(f_{1}, f_{2}\right)$ as follows: given $x^{\prime} \in C$, let $r_{x^{\prime}}$ be the straight path in $B_{x} \approx B^{m}$ joining $x^{\prime}$ to $x$ and define $\tilde{g}\left(x^{\prime}\right)=\left(x^{\prime},\left(f_{1} \circ r_{x^{\prime}}\right) \theta\left(f_{2} \circ r_{x^{\prime}}\right)^{-1}\right)$ by concatenating the resulting image paths in $N$ with the fixed chosen path $\theta$. In view of the Pontryagin-Thom procedure we obtain the well-defined composite map

$$
e_{A}: \pi_{m-1}\left(S^{n-1}\right) \stackrel{E}{\longrightarrow} \pi_{m}\left(S^{n}\right) \longrightarrow \Omega^{\#}\left(f_{1}, f_{2}\right)
$$

where $E$ is the Freudenthal suspension.

Since tubular neighborhoods are essentially unique up to isotopy, the map $e_{A}$ does not really depend on the data $b$ and $\bar{b}$, but only on the resulting orientations of the tangent spaces $T_{x} M$ and $T_{f_{1}(x)} N$. Clearly, changing one of these orientations amounts to 
replacing $e_{A}(\alpha)$ by $e_{A}(-\alpha), \alpha \in \pi_{m-1}\left(S^{n-1}\right)$.Thus, in fact, $e_{A}$ depends only on the co-orientation of $f_{1}$ at $x$, ie on the orientation of the virtual coefficient bundle $\varphi=f_{1}^{*}(T N)-T M$ (cf Conventions 1.15) at $x$ (or, equivalently, of $\tilde{\varphi}=\operatorname{pr}^{*}(\varphi)$ at $(x, \theta), \mathrm{cf}(2-3))$. Furthermore, $e_{A}$ does not really depend on the choice of the element $(x, \theta)$ in $A$ either. Indeed, any path in $A$ (if it projects to a smooth path in $M$ ) gives rise to an isotopy of $B_{x}$ and to the corresponding deformation of the other data needed in our construction.

Step II Given any element $\left\{\alpha_{A_{j}}\right\} \in \oplus \pi_{m-1}\left(S^{n-1}\right)$ in the domain of $e(\operatorname{cf}(3-1))$, the summands $\alpha_{A_{j}}$ are nontrivial for only finitely many path components $A_{1}, \ldots, A_{k}$ of $E\left(f_{1}, f_{2}\right)$. Apply the construction in step I to each of them simultaneously, using disjoint balls $B_{x_{1}}, \ldots, B_{x_{k}}$, and define

$$
e\left(\left\{\alpha_{A_{j}}\right\}\right)=\coprod_{j=1}^{k} e_{A_{j}}\left(\alpha_{A_{j}}\right) \in \Omega^{\#}\left(f_{1}, f_{2}\right) .
$$

If we can (and do!) orient the virtual coefficient bundle $\widetilde{\varphi}$ (cf (2-3)) then the map $e$ is well defined and independent of all other choices (recall our assumption $m \geq 2$ which allows for the necessary disjoint isotopies). In general $e$ is only well defined up to replacing some of the summands $\alpha_{A_{j}}$ by $-\alpha_{A_{j}}$. But in any case the image of $e$ is a well defined subset of $\Omega^{\#}\left(f_{1}, f_{2}\right)$.

Furthermore the construction of $e$ and of its image is compatible with homotopies of $\left(f_{1}, f_{2}\right)$ (cf Proposition 2.1).

Now consider an isolated coincidence point $x \in M$ of $f_{1}$ and $f_{2}$. Identify a neighborhood $U_{y}$ of $y:=f_{1}(x)=f_{2}(x)$ in $N$ with the Euclidean space via a diffeomorphism $\left(U_{y}, y\right) \approx\left(\mathbb{R}^{n}, 0\right)$; also identify $B^{m}$ with a small ball $B_{x} \subset f_{1}^{-1}\left(U_{y}\right) \cap f_{2}^{-1}\left(U_{y}\right)$ around $x$ (as in step I, (i), above) which contains no coincidence point other than $x$. This allows us to define the "index map"

$$
q=\frac{f_{1}-f_{2}}{\left\|f_{1}-f_{2}\right\|}: S^{m-1} \stackrel{\cong}{\stackrel{\longrightarrow}{\longrightarrow}} \partial B_{x} \longrightarrow S^{n-1}
$$

(compare Wyler [25, Theorems 3 and 4]) and its "concentric extension" $Q: B_{x} \rightarrow B^{n}$, $Q(t b(z)):=t q(z)$ for $z \in S^{m-1}$. We can easily deform $f_{1}$ (while leaving it fixed outside of a small neighborhood of $B_{x}$ ) into a map $f_{1}^{\prime}$ such that the corresponding index map $q^{\prime}$ is smooth and $f_{1}^{\prime}=f_{2}+Q^{\prime}$ on $B_{x}$. Indeed, leaving the map $f_{2}$ (which we may assume to be smooth) unchanged, first deform the difference $f_{1}-f_{2}$ in a collar neighborhood of $S^{m-1}$ until $\left(f_{1}-f_{2}\right) \mid S^{m-1}$ coincides with a smooth approximation $q^{\prime}$ of $q$; then use the linear structure on $U_{y} \approx \mathbb{R}^{n}$ to obtain the desired 
homotopy from $\left(f_{1}-f_{2}\right) \mid B_{x}$ to the concentric extension $Q^{\prime}$ of $q^{\prime}$. After a further small homotopy near $x$ we have $f_{1}^{\prime}=f_{2}+Q^{\prime}-\varepsilon \cdot *$ where $* \in S^{n-1}$ is a regular value of $q^{\prime}$. Thus the (generic!) coincidence locus of $f_{1}^{\prime}$ and $f_{2}$ near $x$ consists of the framed submanifold $\varepsilon q^{\prime-1}(\{*\})$ of the $\varepsilon$-sphere around $x$ and hence corresponds to the Freudenthal suspension of the homotopy class of $q^{\prime} \sim q$.

We conclude that the contribution of an isolated coincidence point $x \in C\left(f_{1}, f_{2}\right)$ towards $\omega^{\#}\left(f_{1}, f_{2}\right)$ is given by the image $e_{A}([q])$ of its index map $q$ (cf (3-2) and (3-4); here $A$ contains ( $x$, constant path at $\left.f_{1}(x)=f_{2}(x)\right)$ ).

Theorem 3.1 Assume that the minimum number $M C\left(f_{1}, f_{2}\right)$ is finite. Then the following holds.

(i) If $m \geq 2$, then $\omega^{\#}\left(f_{1}, f_{2}\right)$ lies in the image of the map e (cf $\left.(3-1)\right)$. In particular, if $m>n$ the homology class defined by a generic coincidence manifold $C\left(f_{1}, f_{2}\right)$ in $M$ must vanish.

(ii) If $n \neq 2$, then $M C\left(f_{1}, f_{2}\right) \leq \# \pi_{0}\left(E\left(f_{1}, f_{2}\right)\right)$; more precisely: if $n>2$ and $C\left(f_{1}, f_{2}\right)$ is finite, then there exists a deformation of $f_{1}$ and $f_{2}$ which preserves empty Nielsen classes of coincidence points and replaces each nonempty Nielsen class by (at most) a single coincidence point.

(iii) If $\pi_{m-1}\left(S^{n-1}\right)=0$ (eg if $m>n=1$ or 2 ) then $M C\left(f_{1}, f_{2}\right)=0$, ie $\left(f_{1}, f_{2}\right)$ is homotopic to a coincidence free pair of maps.

Proof We may assume that $C\left(f_{1}, f_{2}\right)$ is finite. Let $x, x_{1}, \ldots, x_{r}$ be the coincidence points whose $\widetilde{g}$-values (cf (1-5)) lie in a given path component $A$ of $E\left(f_{1}, f_{2}\right)$.

Recall that the construction of $\omega^{\#}\left(f_{1}, f_{2}\right)$ involves a generic approximation of $\left(f_{1}, f_{2}\right)$. As a result each isolated coincidence point $x_{j}$ is replaced by an $n$-codimensional coincidence submanifold $C_{j}$ (eg of the form $C_{j}=\varepsilon q_{j}^{\prime-1}(\{*\})$ as in the discussion following (3-4)) which lies in a small neighborhood $U_{j} \approx \mathbb{R}^{m}$ of $x_{j}$ in $M, j=1, \ldots, r$. In order to prove claim (i) we have to "slide" these (disjoint) neighborhoods into a small ball $B_{x} \subset M$ around $x$. For this purpose pick a smooth "Nielsen" path $\sigma_{j}$ from $x_{j}$ to some point $x_{j}^{\prime} \in B_{x}$ such that $\sigma_{j}$ avoids all coincidence points except $x_{j}$. (Here the Nielsen property of $\sigma_{j}$ means that a lifting to $E\left(f_{1}, f_{2}\right), \operatorname{cf}(1-5)$, joins the constant path at $f_{1}\left(x_{j}\right)=f_{2}\left(x_{j}\right)$ to a short path near $\left.f_{1}(x)=f_{2}(x)\right)$. The corresponding arc

$$
a:=\left\{\left(\sigma_{j}(t), t\right) \mid t \in I\right\}
$$

has a tubular neighborhood of the form $a \times \mathbb{R}^{m}$ in $M \times I$. Then the resulting submanifold $a \times C_{j}$ gives rise to a bordism which relates the local $\omega^{\#}$-data of $x_{j}$ (ie the triple $\left(C_{j}, \widetilde{g}_{j}, \bar{g}_{j}^{\#}\right)$ of (partial) coincidence data corresponding to $\left.C_{j}, \mathrm{cf}(1-4)-(1-6)\right)$ to a 
contribution of the form $e_{A}\left(\left[q_{j}\right]\right)$ towards $\omega^{\#}\left(f_{1}, f_{2}\right)$ (compare (3-2) and (3-4)). Since $E$ preserves addition we may iterate our argument to show that all of $\omega^{\#}\left(f_{1}, f_{2}\right)$ lies in the image if $e$.

In order to prove claim (ii) we may have to deform $f_{1}$ and $f_{2}$ (and not just the local $\omega^{\#}$-data). In view of Example 1.3 we need to consider only the case when $m, n \geq 3$. Then, given two isolated coincidence points $x, x_{j}$ in the same Nielsen class, we can join them by a smoothly embedded Nielsen path $\sigma$ which misses all other coincidence points. Similarly $f_{1} \circ \sigma$ will be an embedding after a small deformation of $f_{1}$ in a tubular neighborhood of $\sigma(I)$. Moreover, by making an approximation transverse to $f_{1} \circ \sigma(I)$ and possibly by shifting $I$-levels slightly near finitely many intersection points we obtain a homotopy $F$ from $f_{1} \circ \sigma$ to $f_{2} \circ \sigma$ which leaves endpoints fixed and which coincides with the constant homotopy of $f_{1} \circ \sigma$ only at the initial deformation parameter.

Next identify a small tubular neighbourhood $V \subset M$ of the arc $\sigma(I)$ with $I \times \mathbb{R}^{m-1}$. Without changing anything outside of $V$ we want to use the homotopy $F$ in order to deform $f_{2}$ into a map $f_{2}^{\prime}$ such that $f_{1}$ and $f_{2}^{\prime}$ coincide in $\sigma(I)=I \times\{0\}$ but nowhere else in $V$. Pick a smooth map $\rho: I \rightarrow[0, \infty)$ such that $\rho^{-1}(\{0\})=\{0,1\}$. First deform the identity map of $M$ along the normal rays in $V$ towards $\sigma(I)$ until each point $(t, v)$ of

$$
V_{\rho}:=\left\{(t, v) \in V=I \times \mathbb{R}^{m-1} \mid\|v\| \leq \rho(t)\right\}
$$

gets mapped to $(t, 0)$. Compose this homotopy with $f_{i}, i=1,2$, so that in the end $f_{i}(t, v)=f_{i} \circ \sigma(t)$ whenever $\|v\| \leq \rho(t)$. Now define $f_{2}^{\prime}$ to equal $f_{2}$ outside of $V_{\rho}$, and $f_{2}^{\prime}(t, v):=F\left(t, \frac{\|v\|}{\rho(t)}\right)$ when $(t, v) \in V_{\rho}, t \neq 0,1$ (ie along any normal ray in $V_{\rho}$, starting at $\sigma(t)$, the constant path with value $f_{2}(\sigma(t))$ is replaced by the path $F(t,-)$ from $f_{1}(\sigma(t))$ to $\left.f_{2}(\sigma(t))\right)$.

Clearly $f_{2}^{\prime}$ is homotopic to $f_{2}$. We have replaced our two original coincidence points of $\left(f_{1}, f_{2}\right)$ by the full arc $\sigma(I)$ of coincidence points of $\left(f_{1}, f_{2}^{\prime}\right)$. Extending $\sigma$ to a slightly larger interval $I_{+}=(-\varepsilon, 1+\varepsilon)$ and using suitable tubular neighbourhoods of $\sigma\left(I_{+}\right)$and $f_{1} \circ \sigma\left(I_{+}\right)$we can find sets $B_{x} \subset M$ and $U_{y} \subset N$ as in the discussion of (3-4) such that $\sigma(I) \subset B_{x}$. After further (local) homotopies $f_{1}-f_{2}^{\prime}$ is the concentric extension $Q$ of some index map $q$ (cf (3-4)). Thus in the end $x$ and $x_{j}$ are replaced by just one coincidence point at the center of $B_{x}$. Iterating this procedure we can reduce each Nielsen class to a single point (see [22, Section 3] for the compatibilities of Nielsen decompositions with homotopies of $f_{1}$ and $f_{2}$ ). 
Finally assume that $\pi_{m-1}\left(S^{n-1}\right)$ is trivial. Then at each isolated coincidence point $x$ the index map (cf (3-4)) allows an extension $Q^{\prime}$ without zero. The resulting map $f_{1}^{\prime}=f_{2}+Q^{\prime}$ is homotopic to $f_{1}$ and has no coincidence with $f_{2}$.

Example 3.2 $(m=n \geq 2)$ Here we may identify $\pi_{m-1}\left(S^{n-1}\right)$ with $\mathbb{Z}$ via the mapping degree. Then the map

$$
\begin{array}{ccc}
e: \bigoplus_{A \in \pi_{0}\left(E\left(f_{1}, f_{2}\right)\right)} \mathbb{Z} \longrightarrow \Omega^{\#}\left(f_{1}, f_{2}\right) & =\Omega_{0}\left(E\left(f_{1}, f_{2}\right) ; \tilde{\varphi}\right)=\oplus \mathbb{Z} \oplus \bigoplus \mathbb{Z}_{2} \\
\psi & \omega \\
\omega^{\#}\left(f_{1}, f_{2}\right) & = & \tilde{\omega}\left(f_{1}, f_{2}\right)
\end{array}
$$

is the direct sum of identity maps and mod 2 reductions according to the orientability of the coefficient bundle $\widetilde{\varphi} \mid A$ (compare the discussion following (3-2), and [17, 9.3]). Now assume that $n>2$. After suitable deformations the Nielsen class $C_{A}=\tilde{g}^{-1}(A)$ corresponding to a path component $A$ of $E\left(f_{1}, f_{2}\right)$ consists of at most one point $x_{A}$ (cf Theorem 3.1(ii)).

If $A$ is nonessential we may remove the coincidence at $x_{A}$ altogether. This is clear when $\tilde{\varphi} \mid A$ is oriented since then the index map of $x_{A}$ has degree 0 and hence is nulhomotopic. If $\tilde{\varphi} \mid A$ is not orientable this degree is even; therefore we may replace $x_{A}$ by nearby generic coincidences which occur in pairs of points having the same sign +1 or -1 ; join each such pair by an embedded Nielsen path $\sigma$ which reverses the given local orientation of $\widetilde{\varphi} \mid A$; in a tubular neighbourhood of $\sigma(I)$ the two endpoints have opposite signs and give rise to a nulhomotopic index map (compare the proof of Theorem 3.1(ii)).

We conclude that $M C\left(f_{1}, f_{2}\right) \leq N\left(f_{1}, f_{2}\right)$. In view of Theorem 1.2(iii) we obtain the following classical "Wecken theorem" as a special consequence of Theorem 3.1.

Corollary 3.3 If $m=n \neq 2$, then for all maps $f_{1}, f_{2}: M^{m} \rightarrow N^{n}$ we have

$$
N\left(f_{1}, f_{2}\right)=N^{\#}\left(f_{1}, f_{2}\right)=\operatorname{MCC}\left(f_{1}, f_{2}\right)=\operatorname{MC}\left(f_{1}, f_{2}\right) \text {. }
$$

For $m=n=1$ this follows from Example 1.3.

Remark 3.4 When $m \geq 1$ and $n=1$ or 2 the minimum number $M C\left(f_{1}, f_{2}\right)$ - if it is known to be finite - is rather easily determined (cf Example 1.3 and Theorem 3.1(iii)) except in the dimension setting $m=n=2$ originally studied by $\mathrm{J}$ Nielsen. Here it took 57 years until the central question "are $N\left(f_{1}\right.$, id $)$ and $M C\left(f_{1}\right.$,id $)$ always equal?" was proved by B Jiang $[15 ; 16]$ to have a negative answer. Thus when $m=n=2$ it is not always possible to replace each Nielsen class by at most one coincidence point (compare Theorem 3.1(ii), and Example 3.2). 


\section{The root case}

Let a map $f: M \rightarrow N$ be given. In this section we discuss the Nielsen and minimum numbers of a pair of the form ( $f_{1}=f, f_{2}=$ constant map).

Since $N$ is path-connected these numbers are independent of the constant value of $f_{2}$. Thus, given a basepoint $x_{0} \in M$, put $y_{0}:=f\left(x_{0}\right)$; we may assume that $f_{2}=y_{0}$ (our notation will not distinguish between a constant map and its value).

We define

$$
\begin{aligned}
\operatorname{deg}^{\#}(f) & :=\omega^{\#}\left(f, y_{0}\right) \in \Omega^{\#}\left(f, y_{0}\right), \\
\widetilde{\operatorname{deg}}(f) & :=\widetilde{\omega}\left(f, y_{0}\right) \in \Omega_{m-n}\left(E\left(f, y_{0}\right) ; \widetilde{\varphi}\right), \text { and } \\
\operatorname{deg}(f): & =\omega\left(f, y_{0}\right) \in \Omega_{m-n}\left(M ; \varphi:=f_{1}^{*}(T N)-T M\right)
\end{aligned}
$$

(compare (1-10), Remark 2.4 and (2-3))

According to $[22,2.1]$ we have

$$
\# \pi_{0}\left(E\left(f, y_{0}\right)\right)=\left[\pi_{1}\left(N, y_{0}\right): f_{*}\left(\pi_{1}\left(M, x_{0}\right)\right)\right] .
$$

ie the cardinality of $\pi_{0}\left(E\left(f, y_{0}\right)\right)$ or, equivalently, of the Reidemeister set $R\left(f, y_{0}\right)$, equals the index of the subgroup $f_{*}\left(\pi_{1}\left(M, x_{0}\right)\right)$ in $\pi_{1}\left(N, y_{0}\right)$. Our analysis will be based on the simple but useful observation that the path components of $E\left(f, y_{0}\right)$ can in fact be parametrized by the endpoints of certain liftings in an appropriate covering space of $N$ (cf Corollary 4.2 below).

Consider the commuting diagram

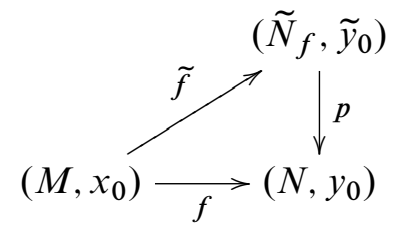

where $p$ denotes the (basically unique) covering such that

$$
p_{*}\left(\pi_{1}\left(\tilde{N}_{f}, \tilde{y}_{0}\right)\right)=f_{*}\left(\pi_{1}\left(M, x_{0}\right)\right)
$$

(cf Greenberg-Harper [11, 6.9]) and $\tilde{f}$ is the lifting of $f$ determined by the choice of $\tilde{y}_{0} \in p^{-1}\left(\left\{y_{0}\right\}\right)$.

Lemma 4.1 For every point $\tilde{y} \in \tilde{N}_{f}$ the space $E(\tilde{f}, \tilde{y})$ is path-connected. 
Proof Since the projection from $E(\tilde{f}, \tilde{y})$ to $M$ is a Hurewicz fibration we have only to join two elements of the form $\left(x_{0}, \tilde{\theta}_{i}\right)$ in $E(\tilde{f}, \tilde{y}), i=1,2$. Now the path $\tilde{\theta}_{1}$ is homotopic $\operatorname{rel}(0,1)$ to a concatenated path $\tilde{\tau} \tilde{\theta}_{2}$ where $\tilde{\tau}$ starts and ends at $\tilde{y}_{0}$. Thus there is a loop $\sigma$ in $M$ starting and ending at $x_{0}$ such that $\tilde{\tau} \sim \tilde{f} \circ \sigma$. Clearly $\sigma$ lifts to a path in $E(\tilde{f}, \tilde{y})$ which joins $\left(x_{0}, \tilde{\theta}_{1}\right)$ to $\left(x_{0}, \tilde{\theta}_{2}\right)$.

The preceding lemma allows us to study our original map $f$ with the help of $\tilde{f}$. Given $(x, \theta) \in E\left(f, y_{0}\right)$, define $\psi(x, \theta)=\tilde{\theta}(1)$ where $\tilde{\theta} \in P\left(\widetilde{N}_{f}\right)$ is the lifting of $\theta \in P(N)$ $(\operatorname{cf}(1-5))$ satisfying $\tilde{\theta}(0)=\tilde{f}(x)$.

Corollary $4.2 \psi$ induces a bijection

$$
\pi_{0}\left(E\left(f, y_{0}\right)\right) \longrightarrow p^{-1}\left(\left\{y_{0}\right\}\right)
$$

which is compatible with homotopies of $f$ (compare [22, 3.2]).

In fact the lifting procedure $\theta \longleftrightarrow \tilde{\theta}$ determined by $\tilde{f}$ yields a homeomorphism

$$
E\left(f, y_{0}\right) \cong \coprod_{\tilde{y} \in p^{-1}\left(\left\{y_{0}\right\}\right)} E(\tilde{f}, \tilde{y}) .
$$

Note that all these path components are homotopy equivalent via concatenation: any path $\tilde{\tau}$ in $\tilde{N}_{f}$ from $\tilde{y}_{1}$ to $\tilde{y}_{2}$ induces a homotopy equivalence

$$
\tilde{\tau}_{\#}: E\left(\tilde{f}, \tilde{y}_{1}\right) \stackrel{\sim}{\longrightarrow} E\left(\tilde{f}, \tilde{y}_{2}\right), \quad \tilde{\tau}_{\#}(x, \tilde{\theta})=(x, \tilde{\theta} \tilde{\tau}) .
$$

Theorem 4.3 Whenever $n \geq 1$ the following statements are equivalent:

(i) $\operatorname{deg}^{\#}(f)=0$;

(ii) $\quad N^{\#}\left(f, y_{0}\right)=0$;

(iii) $N^{\#}\left(f, y_{0}\right) \neq \# \pi_{0}\left(E\left(f, y_{0}\right)\right)$;

(iv) $\operatorname{deg}^{\#}(\tilde{f})=0$;

(v) $N^{\#}\left(\tilde{f}, \tilde{y}_{0}\right)=0$.

In particular, if $\pi_{0}\left(E\left(f, y_{0}\right)\right)$ is infinite or if the manifold $N$ (or $\tilde{N}_{f}$ ) is noncompact, then $\operatorname{deg}^{\#}(f), \operatorname{deg}^{\#}(\tilde{f})$ and the Nielsen numbers $N^{\#}\left(f, y_{0}\right)$ and $N^{\#}\left(\tilde{f}, \tilde{y}_{0}\right)$ vanish.

Remark 4.4 Precisely the analogous statement holds for the ("stabilized") degrees and Nielsen numbers $\widetilde{\operatorname{deg}}(f), \widetilde{\operatorname{deg}}(\tilde{f}), N\left(f, y_{0}\right)$ and $N\left(\tilde{f}, \tilde{y}_{0}\right)$ (cf $[22,1.11]$ ).

In the nonstabilized setting the implication (ii) $\Longrightarrow$ (i) is nontrivial (see Remark 2.3). 
Proof of Theorem 4.3 Clearly (i) $\Longrightarrow$ (ii) $\Longrightarrow$ (iii) and (iv) $\Longleftrightarrow$ (v). Also the full claim of the theorem holds for $N \cong S^{1}$ (cf Example 1.3) and (trivially) for $N \cong \mathbb{R}$.

Thus assume that $n \geq 2$.

In any element $\left[C, \widetilde{g}, \bar{g}^{\#}\right]$ of $\Omega^{\#}\left(f, y_{0}\right)$ or $\Omega^{\#}\left(\tilde{f}, \tilde{y}_{0}\right)$ the homotopy defined by $\widetilde{g}$ induces a vector bundle isomorphism

$$
f^{*}(T N) \mid C \cong \text { const }_{y_{0}}^{*}(T N) \mid C=C \times V
$$

where $V:=T_{y_{0}}(N)$. Thus we may henceforth interpret $\bar{g}^{\#}$ as a trivialization of the normal bundle $v(C, M)$ (compare (1-6)). This gives rise to an identification of a tubular neighborhood $U$ of $C$ in $M$ with $C \times V$.

Now, given path components $A_{1}, \ldots, A_{k}$ and $A$ of $E\left(f, y_{0}\right)$, we construct maps

$$
\Omega^{\#}\left(\tilde{f}, \tilde{y}_{0}\right) \stackrel{\text { pinch }_{\mathscr{A}}}{\longrightarrow} \Omega^{\#}\left(f, y_{0}\right) \stackrel{\text { forg }_{A}}{\longrightarrow} \Omega^{\#}\left(\tilde{f}, \tilde{y}_{0}\right)
$$

as follows.

Let $\left\{\tilde{y}_{1}, \ldots, \tilde{y}_{k}\right\} \subset p^{-1}\left(\left\{y_{0}\right\}\right)$ correspond to $\mathfrak{A}:=\left\{A_{1}, \ldots, A_{k}\right\}$ via $\psi$ (cf Corollary 4.2). Choose pairwise distinct points $z_{i}$ in $V$ as well as paths $\tilde{\tau}_{i}$ in $\tilde{N}_{f}$ from $\tilde{y}_{0}$ to $\tilde{y}_{i}$, $i=1, \ldots, k$. For $c=\left[C, \tilde{g}, \bar{g}^{\#}\right] \in \Omega^{\#}\left(\tilde{f}, \tilde{y}_{0}\right)$ we represent $\operatorname{pinch}_{\mathfrak{A}}(c)$ by the union of the "parallel" submanifolds

$$
C_{i}:=C \times\left\{z_{i}\right\} \subset U
$$

of $M$, together with the data $\widetilde{G}$ and $\bar{G}$ described as follows (compare (1-5) and (1-6)). In order to obtain $\widetilde{G}\left(x, z_{i}\right), x \in C$, apply the covering map $p$ to the concatenated path

$$
\tilde{f}\left(x, z_{i}\right) \stackrel{\tilde{f} \circ \ell}{\sim} \tilde{f}(x) \stackrel{\tilde{g}(x)}{\sim} \tilde{y}_{0} \stackrel{\tilde{\tau}_{i}}{\sim} \tilde{y}_{i}
$$

in $\tilde{N}_{f}$; here $\ell$ denotes the linear path in $\{x\} \times V$ from $\left(x, z_{i}\right)$ to $x=(x, 0) . \bar{G}$ is the obvious trivialization of the normal bundle of $C_{i}$ in $C \times V=U \subset M$, composed with a fixed reflection in $V$ if $T N$ is not orientable along the closed loop $p \circ \tilde{\tau}_{i}$ in $N$.

Similarly, choose a path $\tilde{\tau}$ in $\tilde{N}_{f}$ from $\tilde{y}_{0}$ to $\tilde{y}:=\psi(A) \in p^{-1}\left(\left\{y_{o}\right\}\right)$ (cf Corollary 4.2). The map forg fin in (4) is then built up in three steps. First it forgets all but the $A$-component

$$
\omega_{A}^{\#}=\left[\widetilde{g}^{-1}(A), \widetilde{g}\left|, \bar{g}^{\#}\right|\right]
$$

of an element $\omega^{\#}=\left[C, \widetilde{g}, \bar{g}^{\#}\right] \in \Omega^{\#}\left(f, y_{0}\right)$. Lifting $\tilde{g} \mid$ to $\tilde{N}_{f}$ then makes $\omega_{A}^{\#}$ into an element of $\Omega^{\#}(\tilde{f}, \tilde{y})(\operatorname{cf}(4-4))$. Finally concatenate with $\tilde{\tau}^{-1}$ to obtain $\operatorname{forg}_{A}\left(\omega^{\#}\right) \in$ $\Omega^{\#}\left(\tilde{f}, \tilde{y}_{0}\right)$. 
If $A=A_{i}$ for some $1 \leq i \leq k$ and if the chosen paths $\tilde{\tau}$ and $\tilde{\tau}_{i}$ are homotopic $\operatorname{rel}(0,1)$, then

$$
\operatorname{forg}_{A} \circ \operatorname{pinch}_{\mathfrak{A}}=\text { identity. }
$$

However, if $A \notin \mathfrak{A}=\left\{A_{1}, \ldots, A_{k}\right\}$ then

$$
\text { forg }_{A} \circ \operatorname{pinch}_{\mathfrak{A}} \equiv 0 \text {. }
$$

Let us apply this whole discussion to $\operatorname{deg}^{\#}(f)=\omega^{\#}\left(f, y_{0}\right)$ and $\operatorname{deg}^{\#}(\tilde{f})=\omega^{\#}\left(\tilde{f}, \tilde{y}_{0}\right)$. Identify a small neighborhood $U_{\tilde{y}_{0}}$ of $\tilde{y}_{0}$ in $\tilde{N}_{f}$ with $V$. After a suitable homotopy we may assume that $\tilde{f}$ is smooth, with regular value $\tilde{y}_{0}$, and agrees on a tubular neighborhood $U \cong C \times V$ of $C:=\widetilde{f}^{-1}\left(\left\{\tilde{y}_{0}\right\}\right)$ with the projection to $V=U_{\widetilde{y}_{0}}$.

In the construction of $\operatorname{pinch}_{\mathfrak{A}}(\mathrm{cf}(4-7))$ choose the set $\mathfrak{A}=\left\{A_{1}, \ldots, A_{k}\right\} \subset$ $\pi_{0}\left(E\left(f, y_{0}\right)\right)$ to be so big that $\tilde{f}$ avoids $F^{-}:=p^{-1}\left(\left\{y_{0}\right\}\right)-\left\{\tilde{y}_{1}, \ldots, \tilde{y}_{k}\right\}$. Since $n \geq 2$ we may also choose the paths $\tilde{\tau}_{i}$ to go straight from $\tilde{y}_{0}$ to $z_{i}$ in $U_{\tilde{y}_{o}}=V$, and then to $\tilde{y}_{i}$ via disjoint embedded arcs in $\tilde{N}_{f}-F^{-}, i=1, \ldots, k$.

Now compose $\tilde{f}$ with an isotopy of $\tilde{N}_{f}$ which leaves $F^{-}$fixed and which moves $z_{i}$ along $\tilde{\tau}_{i}$ to $\tilde{y}_{i}$. At the final stage of this deformation the deg ${ }^{\#}$-invariants of $\tilde{f}$ and $f=p \circ \tilde{f}$ satisfy the relation

$$
\operatorname{deg}^{\#}(f)=\operatorname{pinch}_{\mathfrak{A}}\left(\operatorname{deg}^{\#}(\tilde{f})\right)
$$

(cf (4-8)). Thus (iv) $\Rightarrow$ (i) in Theorem 4.3. On the other hand: if $\operatorname{deg}_{A}^{\#}(f)$ is trivial for some path component $A$ of $E\left(f, y_{0}\right)(\operatorname{cf}(2-5))$ then so is

$$
\operatorname{deg}^{\#}(\tilde{f})=\operatorname{forg}_{A}\left(\operatorname{deg}^{\#}(f)\right)
$$

(cf (4-7)). Hence (iii) $\Rightarrow$ (iv) and Theorem 4.3 follows.

Remark 4.5 It follows from (4-10)-(4-14) that for every $A \in \pi_{0}\left(E\left(f, y_{0}\right)\right)$ the triple $\left(C_{A}, \widetilde{g}_{A}, \bar{g}_{A}^{\#}\right)$ (which represents $\operatorname{deg}_{A}^{\#}\left(f, y_{0}\right)$, cf (2-5)) contributes just as much information towards $\operatorname{deg}^{\#}\left(f, y_{0}\right)$ as the full coincidence data $\left(C, \widetilde{g}, \bar{g}^{\#}\right)$ do. In view of Theorem 1.2 and (4-2) this implies the claim concerning the case $\left(f_{1}, f_{2}\right)=(f, *)$ in Theorem 1.4 of the introduction.

Next recall that - due to the compactness of $M$ - our Nielsen numbers are always finite. Thus according to Theorem $1.4 N^{\#}(f, *)$ must vanish if $b(f, *)$ is infinite. It is rather elementary to show even more.

Proposition 4.6 If the index of the subgroup $f_{*}\left(\pi_{1}(M)\right)$ in $\pi_{1}(N)$ is infinite or if $N$ is not compact, then the pair $(f, *)$ is loose; in other words $f$ is homotopic to a map whose image lies in $N-\{*\}$. 
Proof In view of Example 1.3 we may assume that $n \geq 2$. Let $\tilde{f}$ be a lifting of $f$ to the covering space $\tilde{N}_{f}(\mathrm{cf}(4-3))$ which is noncompact here by assumption. Since $M$ is compact $\widetilde{f}(M)$ intersects the fiber $p^{-1}(\{*\})$ in only finitely many points $\widetilde{*}_{1}, \ldots, \widetilde{*}_{k}$. Isotop $\tilde{f}$ along disjoint embedded paths $c_{i}$ in $\tilde{N}_{f}$ which start outside of $\tilde{f}(M)$ and meet the fiber $p^{-1}(\{*\})$ only at the endpoints $c_{i}(1)=\widetilde{*}_{i}, i=1, \ldots, k$. After this homotopy $\tilde{f}$ avoids $p^{-1}(\{*\})$, ie $f=p \circ \tilde{f}$ maps into $N-\{*\}$.

(If $N$ is not compact you may also just compose $f$ with a similar isotopy in $N$ which moves a point $y \in N-f(M)$ to $*$.)

Proof of the root case in Proposition 1.5 $f$ and $\tilde{f}$ allow the same lifting to the common covering space $\tilde{N}_{f}=\tilde{N}_{\tilde{f}}$ (compare (4-3)) of $N$ and $\tilde{N}$. Thus according to Theorem $4.3 N^{\#}(f, *)$ vanishes if and only if $N^{\#}(\tilde{f}, \tilde{*})$ does; otherwise

$$
N^{\#}(f, *)=\left[\pi_{1}(N): f_{*}\left(\pi_{1}(M)\right)\right]=d \cdot\left[\pi_{1}(\tilde{N}): \tilde{f}_{*}\left(\pi_{1}(M)\right)\right]=d \cdot N^{\#}(\tilde{f}, \tilde{*})
$$

and similarly for $N(f, *)$. If $d=\infty$, all these Nielsen numbers vanish.

\section{Selfcoincidences}

In this section we prove the results Theorem 1.4 and Proposition 1.5 as far as selfcoincidences are concerned. Moreover, we relate the selfcoincidence invariant of a map to its degree.

Let $\underline{\Omega}^{\#}\left(f_{1}, f_{2}\right)$ denote the bordism set of triples $\left(C, g, \bar{g}^{\#}\right)$ as in (1-5) (1-6) and (2-1) (without a lifting $\widetilde{g})$. This set is related to $\Omega^{\#}\left(f_{1}, f_{2}\right)$ (cf (2-1)) via the map $p r_{*}$ induced by the projection $p r: E\left(f_{1}, f_{2}\right) \rightarrow M(\mathrm{cf}(1-5))$. In general the resulting coincidence invariant

$$
\underline{\omega}^{\#}\left(f_{1}, f_{2}\right):=p r_{*}\left(\omega^{\#}\left(f_{1}, f_{2}\right)\right)=\left[C\left(f_{1}, f_{2}\right), g, \bar{g}^{\#}\right] \in \underline{\Omega}^{\#}\left(f_{1}, f_{2}\right)
$$

(compare (2-2)) is considerably weaker than $\omega^{\#}\left(f_{1}, f_{2}\right)$ since it captures no longer the Nielsen decomposition of the coincidence set, let alone the other aspects of the lifting $\tilde{g}$.

Now consider a map $f: M \rightarrow N$. The selfcoincidence setting is very special in that $p r$ allows a canonical global section $s: M \rightarrow E(f, f)$ here (defined by $s(x)=(x$, constant path at $f(x)), x \in M)$. We obtain induced maps

$$
\underline{\Omega}^{\#}(f, f) \underset{\mathrm{pr}_{*}}{\stackrel{s_{*}}{\gtrless}} \Omega^{\#}(f, f)
$$


such that $p r_{*} \circ S_{*}=$ identity and clearly

$$
\omega^{\#}(f, f)=s_{*}\left(\underline{\omega}^{\#}(f, f)\right)
$$

(cf (5-1); compare also Proposition 2.6). Thus only the path component of $E(f, f)$ which contains $s(M)$ can possibly be strongly essential. Hence if $\omega^{\#}(f, f) \neq 0$ then $N^{\#}(f, f)=1$. The same argument applies also to the stabilized coincidence invariant $\widetilde{\omega}(f, f)$ (which is precisely as strong as $\omega(f, f)=\operatorname{pr}_{*}(\widetilde{\omega}(f, f))$, compare Koschorke [20; 22]) and $N(f, f)$. In view of Theorem 1.2(iii) this proves the claims of Theorem 1.4 as far as they concern the selfcoincidence case.

As for the proof of Proposition 1.5 just note that $\underline{\omega}^{\#}(\tilde{f}, \tilde{f})=\underline{\omega}^{\#}(f, f)$ whenever $\tilde{f}$ is a lifting of $f$ into any covering space $\tilde{N}$ of $N$. Indeed, the corresponding coincidence data are essentially identical, related by the tangent isomorphism of the covering projection.

Next we show how $\underline{\omega}^{\#}(f, f)$ (and hence $\omega^{\#}(f, f)=s_{*}\left(\underline{\omega}^{\#}(f, f)\right.$, cf $\left.(5-3)\right)$ can be calculated once we know $\left.\operatorname{deg}^{\#}(f)=\omega^{\#}(f, *)\right)$.

Given $k \in \mathbb{Z}$ and $\left[C, \tilde{g}, \bar{g}^{\#}\right] \in \Omega^{\#}(f, *)$, the homotopy $f \mid C \sim *$ described by $\widetilde{g}$ induces a trivialization of $f^{*}(T N) \mid C$ as in (4-6) and, via $\bar{g}^{\#}$, of $v(C, M)$. As in Section 4 we may identify a tubular neighborhood $U$ of $C$ in $M$ with a product $C \times V$ and replace the submanifold $C=C \times\{0\}$ by the union

$$
C(k)=\bigcup C_{i} \subset U \subset M
$$

of $|k|$ "parallel" copies $C_{i}=C \times\left\{z_{i}\right\}$ where the points $z_{i} \in V, i=1, \ldots,|k|$, are pairwise distinct. The obvious linear deformation in $V$ leads to an isomorphism $f^{*}(T N)\left|C \cong f^{*}(T N)\right| C_{i}$; compose it with $\bar{g}^{\#}$ and, if $k$ is negative, with the involution on $C_{i} \times V \cong v\left(C_{i}, M\right) \cong v(C, M)$ determined by a fixed reflection of $V$. We obtain

$$
\bar{g}^{\#}(k): v(C(k), M) \cong f^{*}(T N) \mid C(k) .
$$

Whenever $n \geq 1$ this construction yields a family of canonical maps

$$
\operatorname{pinch}_{k *}: \Omega^{\#}(f, *) \longrightarrow \underline{\Omega}^{\#}(f, f), \quad k \in \mathbb{Z},
$$

which are compatible with homotopies of $f$ and defined by

$$
\operatorname{pinch}_{k *}\left(\left[C, \tilde{g}, \bar{g}^{\#}\right]\right)=\left[C(k) \subset M, \bar{g}^{\#}(k)\right] .
$$

Proposition 5.1 Let $k$ equal the Euler characteristic $\chi(N)$ of $N$ if $N$ is closed and put $k=0$ otherwise. Then for every map $f: M \rightarrow N$ and $* \in N$ we have:

$$
\underline{\omega}^{\#}(f, f)=\operatorname{pinch}_{k *}\left(\omega^{\#}(f, *)\right) .
$$


(The weaker relation $\omega(f, f)=k \operatorname{deg}(f)$ was already proved and applied in [20]).

Proof Identify $V$ with a small neighborhood of $*$ in $N$. After a homotopy we may assume that

(i) $\quad f$ is smooth with regular value $*$, and

(ii) $C(f, *)=f^{-1}(\{*\})$ has a tubular neighborhood $C(f, *) \times V=f^{-1}(V)$ where $f$ is just the projection to $V$.

Now it is possible to choose a smooth vector field on $N$ with generic zeroes $z_{1}, \ldots, z_{|k|}$ which all lie in $V$, and without other zeroes in $f(M)$. Apply the corresponding flow on $N$ to the map $f$ and deform it slightly into a nearby map $f^{\prime}$. Then the coincidence locus $C\left(f^{\prime}, f\right)$ consists of the inverse images $f^{-1}\left(\left\{z_{i}\right\}\right)$ of the fixed points $z_{i}$ of the flow, $i=1, \ldots,|k|$. Clearly the resulting coincidence data represent both $\underline{\omega}^{\#}(f, f)$ and $\operatorname{pinch}_{k *}\left(\omega^{\#}(f, *)\right)$.

Example 5.2 Let $N$ be closed with Euler number $\chi(N) \neq 0$ and commutative nontrivial fundamental group. Given $m>n$, consider the projection

$$
f: M:=N \times S^{m-n} \longrightarrow N
$$

Then $\underline{\omega}^{\#}(f, f)=\operatorname{pinch}_{\chi(N) *}\left(\omega^{\#}(f, *)\right)$ (cf Proposition 5.1) is nontrivial since already the weaker invariant $\omega(f, f)=\chi(N) \cdot \omega(f, *)$ (cf Remark 2.4 and [20, 2.2]) fails to vanish. This can be detected even by singular homology theory (which otherwise is often far too crude to capture coincidence phenomena in higher codimensions). Indeed the composite

$$
\Omega_{m-n}^{f r}(M) \stackrel{\mu}{\longrightarrow} H_{m-n}(M ; \mathbb{Z}) \stackrel{\pi_{2 *}}{\longrightarrow} H_{m-n}\left(S^{m-n} ; \mathbb{Z}\right) \cong \mathbb{Z}
$$

maps $\omega(f, f)$ to $\chi(N)$ (compare the proof of Proposition 5.1. Note that $\varphi=$ $f^{*}(T N)-T M$ is trivial here; $\mu$ and $\pi_{2}$ denote the Hurewicz homomorphism and the second projection, resp.).

Thus according to Theorem 1.4, (1-8), and Theorem 3.1(i)

$$
N(f, f)=N^{\#}(f, f)=M C C(f, f)=1 \quad \neq \quad \# \pi_{0}(E(f, f))=\# \pi_{1}(N)
$$

and $M C(f, f)=\infty$ whenever $n \geq 1$. 


\section{Spherical maps}

In this section we study in detail the special case $M=S^{m}$ Since the minimum numbers $M C$ and $M C C$ as well as the Nielsen numbers are (free) homotopy invariants we may - whenever need be - assume the maps $f_{1}, f_{2}, \ldots$ to have a convenient base point behavior (compare also Appendix A).

First assume $n \geq 2$ so that we can apply Proposition 2.5 to the case where $M=S^{m}$.

Fix basepoints $x_{0} \in S^{m}$ and $y_{0}, y_{1}, y_{2} \in N$ such that $y_{1} \neq y_{2}$, and choose a local orientation of $N$ at $y_{0}$ as well as paths in $N$ joining $y_{0}$ to $y_{1}$ and $y_{2}$. For any two maps $f_{i}:\left(S^{m}, x_{0}\right) \rightarrow\left(N, y_{i}\right), i=1,2$, these choices allow us to identify $\Omega^{\#}\left(f_{1}, f_{2}\right)$ with the homotopy set $\left[S^{m}, S^{n} \wedge\left(\Omega(N)^{+}\right)\right] \approx \pi_{m}\left(S^{n} \wedge\left(\Omega(N)^{+}\right)\right)$where $\Omega(N)$ denotes the space of loops in $N$ starting and ending in $y_{0}$ (cf Proposition 2.5 and its proof). Thus we have a well defined addition both at the level of maps and of $\omega^{\#}$-invariants. We will exploit their compatibilities.

Proposition 6.1 Assume $n \geq 2$. Given $\left[f_{i}\right],\left[f_{i}^{\prime}\right] \in \pi_{m}\left(N, y_{i}\right), i=1,2$, we have

$$
\omega^{\#}\left(f_{1}+f_{1}^{\prime}, f_{2}+f_{2}^{\prime}\right)=\omega^{\#}\left(f_{1}, f_{2}\right)+\omega^{\#}\left(f_{1}^{\prime}, f_{2}^{\prime}\right) .
$$

In particular,

$$
\omega^{\#}\left(f_{1}, f_{2}\right)=\operatorname{deg}^{\#}\left(f_{1}\right)+\omega^{\#}\left(y_{1}, f_{2}\right) .
$$

Furthermore

$$
\operatorname{deg}^{\#}:=\omega^{\#}\left(-, y_{2}\right): \pi_{m}\left(N, y_{1}\right) \longrightarrow \pi_{m}\left(S^{n} \wedge(\Omega N)^{+}\right)
$$

and

$$
\omega^{\#}\left(y_{1},-\right): \pi_{m}\left(N, y_{2}\right) \longrightarrow \pi_{m}\left(S^{n} \wedge(\Omega N)^{+}\right)
$$

are group homomorphisms which determine each other via the group isomorphism inv defined in (the proof of) Proposition 2.5. They measure also the lack of distributivity of $\omega^{\#}, \mathrm{eg}$

$$
\operatorname{deg}^{\#}\left(f_{1}\right)=\omega^{\#}\left(f_{1}, f_{2}\right)+\omega^{\#}\left(f_{1}, f_{2}^{\prime}\right)-\omega^{\#}\left(f_{1}, f_{2}+f_{2}^{\prime}\right) .
$$

Thus (2-12) turns out to be a diagram of homotopy groups and of group homomorphism when $M=S^{m}$ and $n \geq 2$.

Proof The coincidence locus $C\left(f_{1}+f_{1}^{\prime}, f_{2}+f_{2}^{\prime}\right)$ consists of the parts $C\left(f_{1}, f_{2}\right)$ and $C\left(f_{1}^{\prime}, f_{2}^{\prime}\right)$ which lie in disjoint half-spheres of $S^{m}$; moreover the coincidence data $\widetilde{g}$ and $\bar{g}$ are compatible with this decomposition. This establishes the additivity of $\omega^{\#}$. Obvious homotopies such as $\left(f_{1}, f_{2}\right) \sim\left(f_{1}+y_{1}, y_{2}+f_{2}\right)$ and $\left(f_{1}, y_{2}\right) \sim$ $\left(f_{1}+f_{1}-f_{1}, f_{2}+f_{2}^{\prime}-\left(f_{2}+f_{2}^{\prime}\right)\right)$ imply the remaining claims. 
Next let $M N$ stand for any of the numerical homotopy invariants $N, N^{\#}, M C C$ or $M C$ for pairs $\left(f_{1}, f_{2}\right)$ of maps.

Proposition 6.2 Let $m, n \geq 1$. Given $\left[f_{i}\right],\left[f_{i}^{\prime}\right] \in \pi_{m}\left(N, y_{i}\right), i=1,2$, we have the inequality

$$
M N\left(f_{1}+f_{1}^{\prime}, f_{2}+f_{2}^{\prime}\right) \leq M N\left(f_{1}, f_{2}\right)+M N\left(f_{1}^{\prime}, f_{2}^{\prime}\right)
$$

equality holds if $M N\left(f_{1}^{\prime}, f_{2}^{\prime}\right)=0$.

In particular, if $\left[f_{2}\right] \in \pi_{m}^{(2)}\left(N, y_{2}\right)$ (ie if there is an element $\left[\overline{f_{2}}\right] \in \pi_{m}\left(N, y_{1}\right)$ such that $\left(\bar{f}_{2}, f_{2}\right)$ is loose, $\left.c f(1-11)\right)$, then

$$
M N\left(f_{1}, f_{2}\right)=\operatorname{MN}\left(f_{1}-\bar{f}_{2}, y_{2}\right) .
$$

Proof The inequality follows from the decomposition

$$
C\left(f_{1}+f_{1}^{\prime}, f_{2}+f_{2}^{\prime}\right)=C\left(f_{1}, f_{2}\right) \amalg C\left(f_{1}^{\prime}, f_{2}^{\prime}\right) .
$$

Furthermore note that $M N\left(-f_{1}^{\prime},-f_{2}^{\prime}\right)=M N\left(f_{1}^{\prime}, f_{2}^{\prime}\right)$.

Corollary 6.3 If the homomorphism $i_{*}: \pi_{m}(N-\{*\}) \rightarrow \pi_{m}(N)$ (induced by the inclusion) is onto, then $M N\left(f_{1}, f_{2}\right)=0$ for all maps $f_{1}, f_{2}: S^{m} \rightarrow N$, ie $\left(f_{1}, f_{2}\right)$ is loose.

$i_{*}$ is onto eg if $N \neq S^{1}$ has an infinite fundamental group, or if $N$ is not compact, or if $N$ is the product of two manifolds of strictly positive dimensions, or if $N$ fibers over a manifold $B$ such that $\pi_{m}(B-\{*\}) \rightarrow \pi_{m}(B)$ is onto.

Proof After a deformation $f_{1}$ and $f_{2}$ have the correct base point behavior. Then $M N\left(f_{1}, f_{2}\right) \leq M N\left(f_{1}, y_{2}\right)+M N\left(y_{1}, f_{2}\right)=0$.

If $[f] \in \pi_{m}(N)$ and $N=N_{1} \times N_{2}$, deform the two component maps of $f$ until they are constant on opposite half-spheres $S_{ \pm}^{m} \subset S^{m}$; then $f\left(S^{m}\right) \subset N_{1} \vee N_{2} \subset N-\{*\}$. The remainder of our second claim follows from Proposition 4.6 and the homotopy lifting property of fibrations.

Propositions 6.1 and 6.2 allow us also in various other situations to reduce general coincidence questions to the root case (cf Section 4) and, in particular, to a discussion of the degree homomorphism deg $\#$.

Proof of Theorem 1.8 We may replace $f_{1}, f_{2}$ by basepoint preserving maps. In view of symmetry results such as Theorem 1.2(ii) we may also assume the existence of a loose pair $\left(\bar{f}_{2}, f_{2}\right)$ as in Proposition 6.2 and apply Theorem 1.4 to $f=f_{1}-\bar{f}_{2}$. Thus 
$N^{\#}\left(f_{1}, f_{2}\right)=N^{\#}\left(f_{1}-\bar{f}_{2}, y_{2}\right)$ (cf Proposition 6.2) equals the order of $\pi_{1}(N)$ when $m \geq 2$ (and of the cokernel of $f_{1 *}-f_{2 *}$ when $m=n=1$, cf Example 1.3) which must be finite here since $\operatorname{deg}^{\#}\left(f_{1}-\bar{f}_{2}\right)=\omega^{\#}\left(f_{1}, f_{2}\right) \neq 0$ (cf Proposition 4.6).

The calculation of $\operatorname{MCC}\left(f_{1}, f_{2}\right)$ and $M C\left(f_{1}, f_{2}\right)$ follows similarly from Proposition 6.2 and Theorem 1.2(iii). Analogous conclusions are valid for $\widetilde{\omega}\left(f_{1}, f_{2}\right)$ and $N\left(f_{1}, f_{2}\right)$. Our claims are still valid for $n=2$. Indeed, in view of Corollary 6.3, Theorem 1.14, and Theorem 3.1(iii) we have to check only the root case when $m>2$ and $N=S^{2}$ (or $\mathbb{R} P(2))$; after performing a connected sum operation we see that $f^{-1}\left(\left\{y_{2}\right\}\right)$ consists of one (or two "parallel") connected submanifold(s) of $S^{m}$ (cf (4-13)).

How do our minimum numbers behave when $\omega^{\#}\left(f_{1}, f_{2}\right)$ vanishes? Let us focus on this question first in the root case.

Given $m \geq 1$, consider the subgroups (cf Proposition 6.1, Corollary 6.3)

$$
i_{*}\left(\pi_{m}(N-\{*\})\right) \subset \operatorname{ker}\left(\operatorname{deg}^{\#}\right) \quad \text { of } \pi_{m}(N)
$$

represented by maps $f: S^{m} \rightarrow N$ such that $M C(f, *)=M C C(f, *)=0$ and that $N^{\#}(f, *)=0$, resp. (cf Theorem 4.3).

Definition 6.4 $X_{m}(N):=\operatorname{ker}\left(\operatorname{deg}^{\#}\right) / i_{*}\left(\pi_{m}(N-\{*\})\right)$.

This quotient is always an abelian group. If $n \geq 2$ it measures to what extend the sequence

$$
\pi_{m}(N-\{*\}) \stackrel{i_{*}}{\longrightarrow} \pi_{m}(N) \stackrel{\mathrm{deg}^{\#}}{\longrightarrow} \pi_{m}\left(S^{n} \wedge(\Omega N)^{+}\right)
$$

fails to be exact. For a description of $X_{m}(N)$ in terms of pinching maps see Corollary 7.3 below.

Theorem 6.5 (a) Let $Q$ be a smooth connected $q$-manifold and let $p: Q \rightarrow N$ be a smooth locally trivial fibration. Assume that the fiber $F=p^{-1}\left(\left\{y_{0}\right\}\right)$ is compact (and nonempty) and lies in the interior of a smoothly embedded $q$-ball $B$ in $Q$. (This holds, in particular, if $Q$ is a finite covering space over $N$ ). Moreover assume that $q \leq 2 n-2$. Then, given $m \geq 1$, we have: $X_{m}(N)$ vanishes if and only if $X_{m}(Q)$ does.

(b) $X_{m}(N)=0$ in each of the following cases (where $m \geq 1$ is arbitrary unless specified otherwise):

(i) $m \leq 2 n-3$;

(ii) $n \leq 2$ (or $m \leq 3$ );

(iii) $N$ is not compact; 
(iv) $\quad N$ is a sphere $S^{n}$ or a projective space $\mathbb{K} P\left(n^{\prime}\right), \mathbb{K}=\mathbb{R}, \mathbb{C}$ or $\mathbb{U}, n, n^{\prime} \geq 1$;

(v) $N$ is the total space of a Serre fibration with a section and with strictly positive dimensions of the fiber and base space;

(vi) $N$ fibers over a manifold which has an infinite fundamental group.

Proof Example 1.3 establishes these claims whenever $m=1$ or $n=1$. Thus we may assume $m, n \geq 2$ in the remainder of this proof.

Given a smooth fibration $p:\left(Q^{q}, \tilde{y}_{0}\right) \rightarrow\left(N^{n}, y_{0}\right)$, consider the diagram

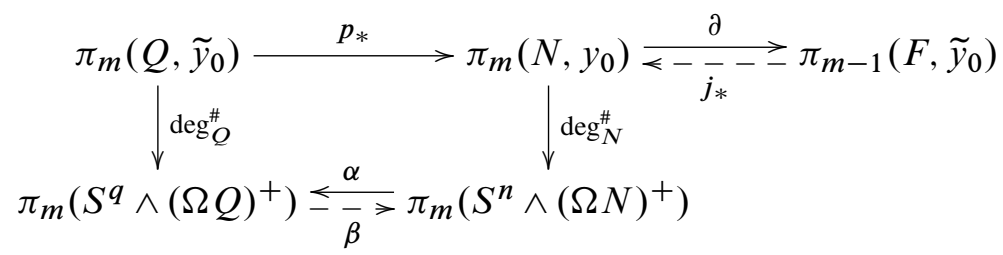

which involves the homotopy sequence of $p$. The homomorphism $\alpha$ is defined as follows. Interpret an element $c \in \pi_{m}\left(S^{n} \wedge(\Omega N)^{+}\right)$- via Pontryagin-Thom - as a bordism class of a framed submanifold $C \subset \mathbb{R}^{m}$, together with a map $\tilde{g}: C \rightarrow \Omega\left(N, y_{0}\right)$. The corresponding evaluation map $C \times I \rightarrow N$ lifts to a homotopy $\widetilde{G}$ in $Q$ from the constant map at $\widetilde{y}_{0}$ to a map $\widetilde{G}_{1}: C \rightarrow F$ which we may assume to be smooth, with regular value $\tilde{y}_{0}$. Endow $C^{\prime}:=\widetilde{G}_{1}^{-1}\left(\left\{\tilde{y}_{0}\right\}\right)$ with the map $\tilde{g}^{\prime}: C^{\prime} \rightarrow \Omega\left(Q, \tilde{y}_{0}\right)$ which corresponds to $\widetilde{G} \mid C^{\prime} \times I$. Moreover compose the natural framing of $C^{\prime}$ in $C$ (given by tangent map of $\widetilde{G}_{1}$ ) with the automorphism of $C^{\prime} \times T_{\widetilde{y}_{0}} F$ which is determined by the homotopy $\widetilde{G} \mid C^{\prime} \times I$ and the tangent bundle along the fibers of $p(\operatorname{cf}[22,3.1])$ The resulting bordism class $\left[C^{\prime} \subset \mathbb{R}^{m}, \tilde{g}^{\prime}\right]$ defines $\alpha(c)$. We have

$$
\operatorname{deg}_{Q}^{\#}=\alpha \circ \operatorname{deg}_{N}^{\#} \circ p_{*}
$$

since $\operatorname{deg}_{N}^{\#} \circ p_{*}$ and $\operatorname{deg}_{Q}^{\#}$ correspond to taking the inverse image of a fiber and of a point in $Q$, resp.

If there exists a basepoint preserving homotopy $\widetilde{J}: F \times I \rightarrow Q$ from the constant map at $\tilde{y}_{0}$ to the fiber inclusion then $p_{\circ} \widetilde{J}$ corresponds to a map $j: F \rightarrow \Omega N$; this induces a splitting of the top line in diagram (6-2) since $\partial \circ j_{*}=$ id. If in addition $q \leq 2 n-2$, ie $\operatorname{dim}(F \times I)<\operatorname{dim} N$, then the image of $j_{*}$ lies already in $i_{*}\left(\pi_{m}\left(N-\{*\}, y_{0}\right)\right)$ for $* \neq y_{0}$.

Finally assume the full hypothesis in Theorem 6.5(a)). Given a framed $q$-codimensional submanifold $C^{\prime} \subset \mathbb{R}^{m}$ together with a map $\tilde{g}^{\prime}: C^{\prime} \rightarrow \Omega\left(Q, \tilde{y}_{0}\right)$, twist the framing via 
the automorphism of $C^{\prime} \times \mathbb{R}^{q}$ determined by $\tilde{g}^{\prime}$ and $T Q$, use the twisted framing to identify a tubular neighbourhood of $C^{\prime}$ in $\mathbb{R}^{m}$ with $C^{\prime} \times B$, reframe the submanifold

$$
C:=C^{\prime} \times F \subset C^{\prime} \times B \subset \mathbb{R}^{m}
$$

using $p, \widetilde{g}^{\prime}$ and a contraction $\widetilde{J}: F \times I \rightarrow B \subset Q$, and equip $C$ with the paths in $N$ which concatenate $p \circ \widetilde{g}^{\prime}$ with the adjoint $j$ of $p \circ \widetilde{J}$. The resulting bordism class $[C, \tilde{g}]$ corresponds to $\beta\left(\left[C^{\prime}, \widetilde{g}^{\prime}\right]\right)$.

This definition of $\beta$ mimics the transition from $\operatorname{deg}_{Q}^{\#}$ to $\operatorname{deg}_{N}^{\#}$ where the inverse image of a point $\tilde{*} \in Q$ is replaced by the inverse image of a whole fiber $p^{-1}(\{*\})$ containing $\widetilde{*}$ (where $* \in N-\left\{y_{0}\right\}$ is close to $y_{0}$ ). The reframings in the construction of $\alpha$ and $\beta$ are motivated by our framing convention in the definition of $\operatorname{deg}^{\#}(\operatorname{cf}(2-10))$. We obtain

$$
\beta \circ \operatorname{deg}_{Q}^{\#}=\operatorname{deg}_{N}^{\#} \circ p_{*}
$$

and $\alpha \circ \beta=\mathrm{id}$. Hence $\beta$ is injective.

Moreover the following three statements are equivalent for every $[f] \in \pi_{m}(Q): f$ can be deformed into the complement of (i) $\{\widetilde{*}\}$, (ii) $\stackrel{\circ}{B}$, (iii) $F$. This completes the proof of claim (a) in Theorem 6.5.

Clearly $X_{m}(N)=0$ whenever $i_{*}$ is onto (eg in case (v) of claim (b); cf Corollary 6.3 and its proof) or when $m<n$. Moreover $X_{m}$ vanishes for all spheres (since deg\# is split injective here, $\mathrm{cf}$ Proposition 2.6) and hence also for all projective spaces (by claim (a)). In particular, $X_{m}(N)=0$ for $N=S^{2}$ or $\mathbb{R} P^{2}$ and also for the remaining surfaces (which are noncompact or have infinite fundamental groups). This settles case (ii) of Theorem 6.5(b). Case (i) is a (weak) consequence of [22, Theorem 1.10].

Remark 6.6 In the previous discussion we have been dealing with the very special case where $M$ is a sphere and $f_{2}$ is constant. Here no problem arises in the dimension setting $m=n=2$ which is so critical in classical fixed point theory: see Jiang [16] for examples where $N(f, \mathrm{id})=N^{\#}(f, \mathrm{id})=0<M C C(f, \mathrm{id}) \leq M C(f, \mathrm{id})$.

Example 6.7 (Stiefel manifolds and Grassmannians) (a) Given integers $1 \leq k<r$, let $p_{r, k}: V_{r, k} \rightarrow G_{r, k}$ be the fiber projection which maps each orthonormal $k$-frame in $\mathbb{R}^{r}$ to the $k$-plane it spans. Assume $2 k \leq r$. (This is no restriction as far as Grassmannians are concerned since $\left.G_{r, k} \cong G_{r, r-k}\right)$. Then the fiber $O(k)=V_{k, k}$ of $p_{r, k}$ can be deformed into a ball via the isotopy

$$
V_{k, k} \times\left[0, \frac{\pi}{2}-\varepsilon\right] \longrightarrow V_{r, k},
$$


$\left(\left\{v_{i}\right\}, t\right) \rightarrow\left\{\cos (t) v_{i}+\sin (t) e_{k+i}\right\}$, where $1 \leq i \leq k$ and $e_{j}$ denotes the $j^{\text {th }}$ standard unit vector in $\mathbb{R}^{r}$. Thus, given $m \geq 1$, we conclude from Theorem 6.5(a)) that $X_{m}\left(G_{r, k}\right)=0$ if and only if $X_{m}\left(V_{r, k}\right)=0$.

(b) In turn, if the sphere $S^{r-1}$ allows $k-1$ linearly independent vector fields then the canonical fibration $V_{r, k} \rightarrow S^{r-1}$ has a section and $X_{m}\left(V_{r, k}\right)=0$ by Theorem $6.5(\mathrm{~b})(\mathrm{v})$.

We conclude for instance that $X_{m}\left(G_{r, k}\right)=0$ for all $m \geq 1$ and $1 \leq k<r$ if $r=2,4,8$, or 16 (compare eg Atiyah-Bott-Shapiro [1, page 38]).

The same statements hold for the Grassmann manifold $\widetilde{G}_{r, k}$ of oriented $k$-planes in $\mathbb{R}^{r}$ (with the added advantage that no map into $\widetilde{G}_{r, k}$ is coincidence producing).

Remark 6.8 (and proof of Theorem 1.11) The homogeneity techniques supplied by Propositions 6.1 and 6.2 allow us (in analogy to the proof of Theorem 1.8) to extend the condition " $X_{m}(N)=0$ " to yield the following equivalent condition: "For every pair of maps $f_{1}, f_{2}: S^{m} \rightarrow N$ which are not both coincidence producing we have: $\left(f_{1}, f_{2}\right)$ is loose if and only if $\omega^{\#}\left(f_{1}, f_{2}\right)=0 "$.

Clearly this implies Theorem 1.11 of the introduction.

Proof of the statements in Example 1.12 In view of Example 1.3 we may assume that $n \geq 2$. In the case $N=S^{n} \mathrm{deg}^{\#}$ is split injective (cf Proposition 2.6) and $X_{m}(N)=0$ (cf Theorem 6.5). Moreover $\left(f_{1}, f_{2}\right)$ is loose if and only if $f_{1} \sim a f_{2}$ (cf Dold-Gonçalves [8, 1.10] or Koschorke [22, Section 8]). Thus it follows from Theorem 1.8 and Theorem 1.11 that $N^{\#}\left(f_{1}, f_{2}\right)=\operatorname{MCC}\left(f_{1}, f_{2}\right)$ equals 0 or $\# \pi_{0}\left(E\left(f_{1}, f_{2}\right)\right)$ according as $\omega^{\#}\left(f_{1}, f_{2}\right)=\operatorname{deg}^{\#}\left(f_{1}-a \circ f_{2}\right)$ (cf Proposition 6.1) vanishes or not. The restriction $n \neq 2$ in Theorem 1.8 can be avoided here by a connected sum argument applied to the (generically) framed manifold $\left(f_{1}-a \circ f_{2}\right)^{-1}\{*\}$ (compare Proposition 2.6).

If $M C\left(f_{1}, f_{2}\right)$ is finite and $m>n \geq 2$ then $\operatorname{deg}^{\#}\left(f_{1}-a \circ f_{2}\right)=e(\beta)$ for some $\beta \in$ $\pi_{m-1}\left(S^{n-1}\right)$ (cf Theorem 3.1) and hence in view of Proposition $2.6\left[f_{1}-a \circ f_{2}\right]=$ $\operatorname{coll}_{*}\left(\left[f_{1}-a \circ f_{2}\right]\right)= \pm E(\beta)$.

Before we discuss the minimum number $M C\left(f_{1}, f_{2}\right)$ for maps into a general target manifold $N$ we introduce the composite homomorphism (for $m>2 \leq n$ )

(6-5) $\eta: \bigoplus_{A \in \pi_{1}(N)} \pi_{m-1}\left(S^{n-1}\right) \longrightarrow \pi_{m-1}\left(\bigvee_{A} S^{n-1}\right) \stackrel{\left.v\right|_{*}}{\longrightarrow} \pi_{m-1}(N-\{*\})$

as follows. Choose a homeomorphism $u$ from the unit ball $B^{n} \subset \mathbb{R}^{n}$ onto a small ball $B(*)$ in $N$ centered at $*$ such that $y_{0}=u\left(x_{0}\right) \in \partial B(*)$ (where $x_{0} \in S^{n-1}$ 
is a basepoint); lift $u$ in the universal cover $\tilde{N}$ to all the various levels (which are parametrized by $\left.A \in \pi_{1}\left(N, y_{0}\right) \approx \pi_{0}\left(E\left(f_{1}, f_{2}\right)\right)\right)$; join the lifted basepoints $\left\{\tilde{u}_{A}\left(x_{0}\right)\right\}$ by appropriate paths to $\tilde{y}_{0}$ and project back to $N$. We obtain the map

$$
v:\left(\bigvee_{A} B^{n}, \bigvee_{A} S^{n-1}\right) \longrightarrow(N, N-\{*\})
$$

Compose the induced homomorphism $\left.v\right|_{*}$ with the natural inclusion to define $\eta$ in $(6-5)$.

On the other hand consider the maps

$$
\bigvee_{A \in \pi_{1}(N)} S^{n} \underset{\text { ret }}{\stackrel{\text { in }}{\rightleftarrows}} S^{n} \wedge\left(\Omega N^{+}\right)=\operatorname{Thom}\left(\Omega N \times \mathbb{R}^{n}\right)
$$

corresponding (at the level of Thom spaces of trivial $n$-plane bundles) to the obvious componentwise inclusion and retraction maps between $\pi_{1}(N)=\pi_{0}(\Omega N)$ and $\Omega N$. We obtain the diagram (for $m \geq 2$ )

$$
\bigoplus_{A \in \pi_{1}(N)} \pi_{m}\left(S^{n}\right) \subset \pi_{m}\left(\bigvee_{A} S^{n}\right) \underset{\mathrm{ret}_{*}}{\stackrel{\mathrm{in} *}{\gtrless}} \pi_{m}\left(S^{n} \wedge(\Omega N)^{+}\right)
$$

where ret $_{*} \circ \mathrm{in}_{*}=$ identity. Moreover, $\mathrm{in}_{*}$ and the Freudenthal suspension compose to yield the homomorphism

$$
\pm e=\mathrm{in}_{*} \mid \circ \oplus E: \bigoplus_{A \in \pi_{1}(N)} \pi_{m-1}\left(S^{n-1}\right) \longrightarrow \pi_{m}\left(S^{n} \wedge(\Omega N)^{+}\right)
$$

constructed at the beginning of Sections 3 and 6.

Theorem 6.9 Assume $m \geq 3$. Given any pair of maps $f_{1}, f_{2}: S^{m} \rightarrow N$ such that $f_{2}$ is not coincidence producing, we have:

If $M C\left(f_{1}, f_{2}\right)<\infty$ then $\omega^{\#}\left(f_{1}, f_{2}\right) \in e(\operatorname{ker}(\eta))$. In turn, if $X_{m}(N)=0$ and $\omega^{\#}\left(f_{1}, f_{2}\right) \in e(\operatorname{ker}(\eta))$ then $\operatorname{MC}\left(f_{1}, f_{2}\right)$ is finite.

Proof In view of Propositions 6.1 and 6.2 we need to consider only pairs of the form $(f, *)$. Interpret $f$ as a map from $I^{m}$ to $N$ which maps the boundary $\partial I^{m}$ to the basepoint $y_{0} \neq *$. If $M C(f, *)<\infty$ then after a deformation $f^{-1}(\{*\})$ consist of finitely many points $x_{j} \in I^{m}, j=1, \ldots, k$. Furthermore there are small balls $B_{x_{j}} \subset \stackrel{\circ}{I}^{m}$ centered at $x_{j}$ such that $f$ maps $\partial B_{x_{j}} \cong S^{m-1}$ to the boundary sphere of the 
ball $B(*)$ around $*$ in $N$. After isotoping $x_{j}$ into suitable positions in $I^{m}=I^{m-1} \times I$ we may even assume that

$$
\begin{aligned}
& f\left(I^{m-1} \times\left[0, \frac{1}{2}\right]\right) \subset v\left(\bigvee_{A} B^{n}\right) \quad(\operatorname{cf}(6-6)) \quad \text { and } \\
& f\left(I^{m-1} \times\left[\frac{1}{2}, 1\right]\right) \subset N-\stackrel{\circ}{B}(*) .
\end{aligned}
$$

Thus the index maps of the coincidence points of $(f, *)(\operatorname{cf}(3-4))$ determine an element $\alpha \in \operatorname{ker} \eta$ (since $f \mid I^{m-1} \times\left\{\frac{1}{2}\right\}$ is nulhomotopic in $N-\{*\}$ ) such that $e(\alpha)=\omega^{\#}(f, *)$. On the other hand, every $\alpha \in \operatorname{ker}(\eta)$ and a corresponding nulhomotopy in $N-\{*\}$ yields a map $f^{\prime}: S^{m} \rightarrow N$ with $M C\left(f^{\prime}, *\right)<\infty$ and $\omega^{\#}\left(f^{\prime}, *\right)=e(\alpha)$. If $\omega^{\#}(f, *)=e(\alpha)$ and $X_{m}(N)$ vanishes then so do $\operatorname{deg}^{\#}\left(f-f^{\prime}\right)$ and hence $M C\left(f-f^{\prime}, *\right)$.

Since $M C(f, *) \leq M C\left(f^{\prime}, *\right)+M C\left(f-f^{\prime}, *\right)$ we conclude that this minimum number is finite.

We illustrate our criterion by a sample application.

Corollary 6.10 Let $N=S^{n} / G$ be a spherical space form (compare Example 1.13) and assume $m, n \geq 2$. Then we have for all $[f] \in \pi_{m}(N)$

$$
M C(f, *)<\infty \Longleftrightarrow[\tilde{f}] \in \begin{cases}E(\operatorname{ker} h) & \text { if } \# \geq 3 ; \\ E\left(\pi_{m-1}\left(S^{n-1}\right)\right) & \text { if \# } \leq \leq 2 .\end{cases}
$$

Here $[\tilde{f}] \in \pi_{m}\left(S^{n}\right)$ is obtained by lifting $[f], E$ denotes the Freudenthal suspension and

$$
h:=\bigoplus_{j=0}^{\infty} h_{j}: \pi_{m-1}\left(S^{n-1}\right) \longrightarrow \pi_{m-1}\left(S^{2 n-3}\right) \oplus \pi_{m-1}\left(S^{3 n-5}\right) \oplus \ldots
$$

is the (total) Hopf-Hilton homomorphism (cf Whitehead [24, XI, 8.5].

Recall that $E\left(\pi_{m-2}\left(S^{n-2}\right)\right)$ lies in the kernel of $h$ whenever $n \geq 3$ (and coincides with it if also $m \leq 3 n-6$; see, for example, [24, XII, 2.3] or Koschorke-Sanderson [23]).

Proof If $n$ is even, then $\chi\left(S^{n}\right)=2 \geq \# G$. In particular, this implies our claim when $m=n=2$.

Thus (in view of Theorem 3.1(iii)) we may assume that $m, n \geq 3$. Then $\eta$ (cf (6-5)) lifts to

$\tilde{\eta}:\left(\pi_{m-1}\left(S^{n-1}\right)\right)^{k} \subset \pi_{m-1}\left(\bigvee^{k} S^{n-1}\right) \stackrel{\left.\widetilde{v}\right|_{*}}{\longrightarrow} \pi_{m-1}\left(S^{n}-\left\{\tilde{*}_{1}, ., \tilde{*}_{k}\right\}\right) \cong \pi_{m-1}\left(\bigvee^{k-1} S^{n-1}\right)$ 
where $k:=\# \pi_{1}(N)=\# G$ and the points $\widetilde{*}_{1}, \ldots, \widetilde{*}_{k} \in S^{n}$ are projected to $* \in N$. After a suitable homotopy $v \mid \bigvee^{k-1} S^{n-1}$ (cf (6-6)) lifts to an embedding

$$
\tilde{v} \|: \bigvee^{k-1} S^{n-1} \subset \mathbb{R}^{n}-\left\{\widetilde{*}_{1}, \ldots, \widetilde{*}_{k-1}\right\} \approx S^{n}-\left\{\widetilde{*}_{1}, \ldots, \widetilde{*}_{k}\right\}
$$

which is known to be a homotopy equivalence. We use this to identify the target group of $\tilde{\eta}$ with $\pi_{m-1}\left(\bigvee^{k-1} S^{n-1}\right)$ and thus make it accessible to Hilton's computing techniques [13].

Let $\iota_{j} \in \pi_{n-1}\left(\bigvee S^{n-1}\right)$ be represented by the inclusion of the $j$ th sphere in the wedge. Then

$$
\begin{aligned}
\left.\widetilde{v}\right|_{*}\left(\iota_{j}\right) & =\iota_{j}, \quad j=1, \ldots, k-1, \text { and } \\
\left.\widetilde{v}\right|_{*}\left(\iota_{k}\right) & =-\iota_{1}-\cdots-\iota_{k-1} ;
\end{aligned}
$$

indeed, we can retract the boundary sphere of a ball around the point $\widetilde{*}_{k}=\infty \in \mathbb{R}^{n} \cup\{\infty\}$ into the spheres around the remaining points $\widetilde{*}_{1}, \ldots, \widetilde{*}_{k-1}$, reversing orientations in the process. Let $r$ stand for a suitable reflection (ie selfmap of degree -1 ) on any sphere.

Now consider an element $\alpha=\left(\alpha_{1}, \ldots, \alpha_{k}\right)$ in the domain of $\tilde{\eta}$. When $k=2$ then $\eta(\alpha)=0$ if and only if $\alpha_{2}=-r_{*}\left(\alpha_{1}\right)$; in this case $E\left(\alpha_{1}\right)=E\left(\alpha_{2}\right)$. When $k=3$ then $\eta(\alpha)$ vanishes if and only if

$$
\left(\iota_{1}+\iota_{2}\right) \circ \alpha_{3}=\iota_{1} \circ \alpha_{3}+\iota_{2} \circ \alpha_{3}+\sum_{j \geq 0} w_{j}\left(\iota_{1}, \iota_{2}\right) \circ h_{j}\left(\alpha_{3}\right)
$$

(cf Whitehead [24, XI, 8.5]) equals $-\left(-\iota_{1}\right) \circ \alpha_{1}-\left(-\iota_{2}\right) \circ \alpha_{2}$, or, equivalently, $\alpha_{1}=$ $\alpha_{2}=\alpha_{3} \in \operatorname{ker} h$. (In order to see this, project to each of the wedge factors $S^{n-1}$ and permute their roles; note also that $\operatorname{ker} h \subset \operatorname{ker}\left(r_{*}+\mathrm{id}\right):$ just substitute $\iota_{1}$ and $\iota_{2}$, resp., by $r$ and id, resp., in (6-10)). Collapsing all but three spheres in the wedge $\bigvee S^{n-1}$ allows us to extend our calculation of $\operatorname{ker} \eta=\operatorname{ker} \tilde{\eta}$ also to the case where $k>3$.

Next observe that $X_{m}(N)=0$ (cf Theorem 6.5(a) and (b)(iv)). Thus according to Theorem 6.9 $M C(f, *)$ is finite if and only if $\operatorname{deg}^{\#}(f) \in e(\operatorname{ker} \eta)$ (cf (6-8)) or, equivalently, $\operatorname{deg}^{\#}(\tilde{f})$ lies in the image, under $\operatorname{in}_{\ell *} \circ E$ for some suitable loop $\ell \in \Omega(N)$ (cf (2-12)), of $\pi_{m-1}\left(S^{n-1}\right)$ and of ker $h$, resp. (see Remark 4.5). But this yields the indicated condition concerning the homotopy class $[\tilde{f}]=\operatorname{coll}_{*}([\tilde{f}]$ ) (cf Proposition 2.6).

Example 6.11 Let $N=S^{3} / G$ be a 3-dimensional spherical space form and $m=4$. Then $h: \pi_{3}\left(S^{2}\right) \stackrel{\cong}{\longrightarrow} \mathbb{Z}$ and $E$ maps this group onto $\pi_{4}\left(S^{3}\right) \cong \pi_{4}(N) \cong \mathbb{Z}_{2}$. 
Hence according to Theorem 1.4, Proposition 2.6, Theorem 4.3, and Corollary 6.10 we have for every map $f: S^{4} \rightarrow N$

$$
M C(f, *)= \begin{cases}\infty & \text { if }[f] \neq 0 \text { and } \# G \geq 3 \\ \# G & \text { if }[f] \neq 0 \text { and } \# G \leq 2 \\ 0 & \text { if }[f]=0\end{cases}
$$

In particular, if $\# G \geq 3$ and $[f] \neq 0$ then

$$
\operatorname{MC}(f, *)=\infty \quad \text { but } \operatorname{MC}(\tilde{f}, \tilde{*})=1
$$

where $\tilde{f}: S^{4} \rightarrow S^{3}$ is a lifting of $f$.

In order to gain a better geometric understanding of the dependence on $\# G$ which we encountered in Corollary 6.10 and in the last example let us recall (and generalize) a classical notion (cf Wyler [25, page 29]).

Definition 6.12 Given a map $f: M \rightarrow N$ we call a point $y \in N$ injective (or almost injective, resp.) if its inverse image $f^{-1}(\{y\})$ consists of a single point (or is finite, resp.).

Thus $M C(f, *)<\infty$ if and only if $f$ is homotopic to a map which possesses an almost injective point. Furthermore, given a lifting $\tilde{f}$ of $f$ to a $k$-sheeted covering space $\tilde{N}$ over the manifold $N, M C(f, *)<\infty$ if and only if $\tilde{f}$ is homotopic to a map which has at least $k$ different almost injective points (which we may isotop into the fiber $\left\{\widetilde{*}_{1}, \ldots, \widetilde{*}_{k}\right\}$ over $\left.* \in N\right)$.

Clearly if a map between spheres is an (unreduced) suspension then it has a least two injective points. Suspending again, we obtain a map which admits a whole circle of injective points. In Corollary 6.10 and Example 6.11 Hopf-Hilton invariants describe the precise borderline between single and double suspensions which characterizes spherical maps having at least as many almost injective points as there are elements in $G$.

Proof of the statements in Example 1.13 Since $n$ is odd $N$ admits a nowhere vanishing vector field. Hence for every map $f$ into $N$ the pair $(f, f)$ is loose. In particular if $f_{1} \not f_{2}$ and $m>1$ then $\omega^{\#}\left(f_{1}, f_{2}\right)=\operatorname{deg}^{\#}\left(f_{1}-f_{2}\right)$ (cf Proposition 6.1) is nontrivial since $X_{m}(N)=0(\operatorname{cf}$ Theorem 6.5(a) and (b)) and we see from a lifting argument that $f_{1}-f_{2}$ does not map into $N-\{*\}$. The statements in Example 1.13 follows from Theorem 1.8 and Corollary 6.10 (see also Example 1.3). 
Remark 6.13 The approach of the preceding proof still works when $n$ is even provided $f_{2}$ occurs in a loose pair $\left(\bar{f}_{2}, f_{2}\right)$ (so that we may replace $\left(f_{1}, f_{2}\right)$ by $\left(f_{1}-\bar{f}_{2}, *\right)$, cf Propositions 6.1 and 6.2). But this proviso can be very restrictive. For instance if $N$ is an even-dimensional real projective space and $m \geq 2$, then it implies that both liftings $\tilde{f}_{2}$ and $a \circ \tilde{f}_{2}$ of $f_{2}$ in $S^{n}$ must be homotopic to $a \circ \widetilde{\bar{f}}_{2}$ where $a$ denotes the antipodal map (cf Dold-Gonçalves [8, 1.10]). Thus $\tilde{f}_{2} \sim a \circ \widetilde{f}_{2}$ and, if $\left[\tilde{f}_{2}\right] \in E\left(\pi_{m-1}\left(S^{n-1}\right)\right)$, then $2\left[f_{2}\right]=0$. This restriction is satisfied, for example, by only two elements in $\pi_{17}(\mathbb{R} P(10)) \cong \pi_{17}\left(S^{10}\right)=E\left(\pi_{16}\left(S^{9}\right) \cong \mathbb{Z}_{240}\right.$.

Next consider again an arbitrary manifold $N$ as well as a pair of maps $f_{1}, f_{2}: S^{m} \rightarrow N$, $m \geq 2$, with only finitely many coincidence points. Adding up their index maps (cf (3-4)) while keeping track of the Nielsen decomposition we obtain the "total index"

$$
\operatorname{ind}\left(f_{1}, f_{2}\right)=\left\{\operatorname{ind}_{A}\left(f_{1}, f_{2}\right)\right\} \in \bigoplus_{A \in \pi_{1}(N)} \pi_{m-1}\left(S^{n-1}\right)
$$

which is well defined (once the choices of an orientation of $N$ at $y_{0}$ and of paths joining $y_{0}$ to $f_{1}\left(x_{0}\right)$ and $f_{2}\left(x_{0}\right)$ have been fixed as at the beginning of this section).

Theorem 6.14 The suspended index

$$
\bigoplus E\left(\operatorname{ind}\left(f_{1}, f_{2}\right)\right) \in \bigoplus_{A \in \pi_{1}(N)} \pi_{m}\left(S^{n}\right)
$$

is determined by $\omega^{\#}\left(f_{1}, f_{2}\right)$ and hence depends only on the basepoint preserving homotopy classes of $f_{1}$ and $f_{2}$.

Furthermore, if the suspension $E: \pi_{m-1}\left(S^{n-1}\right) \rightarrow \pi_{m}\left(S^{n}\right)$ is injective then

$$
M C\left(f_{1}, f_{2}\right)=N^{\#}\left(f_{1}, f_{2}\right)=\#\left\{A \in \pi_{1}(N) \mid \operatorname{ind}_{A}\left(f_{1}, f_{2}\right) \neq 0\right\} .
$$

Proof When we apply the monomorphism in in $_{*}(\mathrm{cf}(6-7))$ to the suspended index we obtain $e\left(\operatorname{ind}\left(f_{1}, f_{2}\right)\right)=\omega^{\#}\left(f_{1}, f_{2}\right)(\mathrm{cf}(6-8)$ and the discussion preceding Theorem 3.1).

If $n>2$ we may replace any Nielsen class by (at most) one single point $x$ (cf Theorem 3.1(ii)); if it is nonessential we may remove it altogether provided $E$ is injective and hence the index map $q$ at $x$ (cf Equation (3-4)) is nulhomotopic. In view of Theorem 3.1(iii), Proposition 6.2, Corollary 6.3, and (the last statement in) Proposition 2.6 it remains only to consider the case where $m=2$ and $N=\mathbb{R} P(2)$. But here our second claim follows from Jezierski [14, Theorem 4.0]. (I am grateful to S Bogaty̆ and E Kudryavtseva for bringing this reference to my attention). 


\section{Hopf-Ganea invariants and the degree deg $^{\#}$}

In this section we continue our discussion of the case $M=S^{m}, m \geq 2$. We give a purely homotopy theoretical description of our (geometric) homomorphism deg and of the group $X_{m}(N) . \mathrm{deg}^{\#}$ turns out to consist of two components, one of them being a Hopf-Ganea invariant $H_{\mathcal{C}}$. We show that $H_{\mathcal{C}}$ is a finiteness obstruction for the minimum number $M C$ (and actually the only one in a dimension range depending on the connectivity of $N$ ).

Assume that $k:=\# \pi_{1}(N)$ is finite (otherwise $\operatorname{deg}^{\#} \equiv 0$, cf Proposition 4.6). Let $p: \tilde{N} \rightarrow N$ be the universal covering of $N$ and let $\bigvee B^{n}$ denote a wedge of standard unit $n$-balls, pinched together at base points of the boundary spheres. Given points $y_{0} \neq * \in N$ and $\tilde{y}_{0} \in p^{-1}\left(\left\{y_{0}\right\}\right)$ as well as an orientation of $\tilde{N}$, we can identify $\bigvee^{k} B^{n}$ with a union of (compact) balls around $\widetilde{*}_{1}, \ldots, \widetilde{*}_{k} \in p^{-1}(\{*\})$ in $\tilde{N}$ which intersect at $\tilde{y}_{0}$. We obtain a pinching map

$$
\text { pinch: } \tilde{N}=\left(\tilde{N}-\cup^{k} \stackrel{\circ}{B^{n}}\right) \cup_{\text {att }} \bigvee^{k} B^{n} \longrightarrow \tilde{N} / \bigvee^{k} S^{n-1} \cong \bigvee^{k} S^{n} \vee \tilde{N}
$$

which collapses the boundary spheres $\bigvee S^{n-1}$ to a point.

On the other hand there is the projection

$$
\operatorname{proj}_{2}: \bigvee^{k} S^{n} \vee \tilde{N} \longrightarrow \tilde{N}
$$

which collapses the wedge $\bigvee^{k} S^{n}$. Its mapping fiber (cf Whitehead [24, page 43] but without a retopologization as in [24, page 20, line 7]).

$$
F=\left\{(y, \theta) \in\left(\bigvee^{k} S^{n} \vee \tilde{N}\right) \times P(\tilde{N}) \mid \theta(0)=\operatorname{proj}_{2}(y), \theta(1)=\tilde{y}_{0}\right\}
$$

(compare Conventions 1.15) contains the contractible subspace

$$
P=\{(y, \theta) \in F \mid y \in \tilde{N}\} \cong\left\{\theta \in P(\tilde{N}) \mid \theta(1)=\tilde{y}_{0}\right\}
$$

whose complement $\left(S^{n}-\left\{\tilde{y}_{0}\right\}\right) \times\left(\amalg_{k} \Omega\left(\tilde{N}, \tilde{y}_{0}\right)\right)$ admits a homotopy equivalence with $\left(S^{n}-\left\{\tilde{y}_{0}\right\}\right) \times \Omega\left(N, y_{0}\right)$ which is compatible with the labelling of the center points $\tilde{*}_{1}, \ldots, \tilde{*}_{k} \in p^{-1}(\{*\})$ of the balls in $\bigvee^{k} B^{n}$.

Lemma 7.1 (cf Cornea [6, page 2769]) The quotient map

$$
\text { quot: } F \longrightarrow F / P \cong S^{n} \wedge\left(\Omega\left(N, y_{0}\right)^{+}\right)
$$

is a homotopy equivalence. 
Proof Let $\theta_{0}$ denote the constant loop at $\tilde{y}_{0}$. We describe a map

$$
\left(\left(\bigvee^{k} S^{n}\right) \times \Omega\left(\tilde{N}, \tilde{y}_{0}\right),\left\{\tilde{y}_{0}\right\} \times \Omega\left(\tilde{N}, \tilde{y}_{0}\right)\right) \longrightarrow\left(F,\left\{\left(\tilde{y}_{0}, \theta_{0}\right)\right\}\right)
$$

which induces the required homotopy inverse. In each sphere $S^{n}$ contract all points $y$ with $\operatorname{dist}\left(y, \tilde{y}_{0}\right)=1$ into the wedge point $\tilde{y}_{0}$ and use this distance also as a parameter for how far to deform loops $\theta \in \Omega\left(\tilde{N}, \tilde{y}_{0}\right)$ along themselves. Thus at $y=\tilde{y}_{0}$ each loop is fully retracted to $\theta_{0}$ while $\theta$ is left unchanged if $\operatorname{dist}\left(y, \tilde{y}_{0}\right) \geq 1$.

The maps in (7-1), (7-2), and Lemma 7.1 yield the diagram

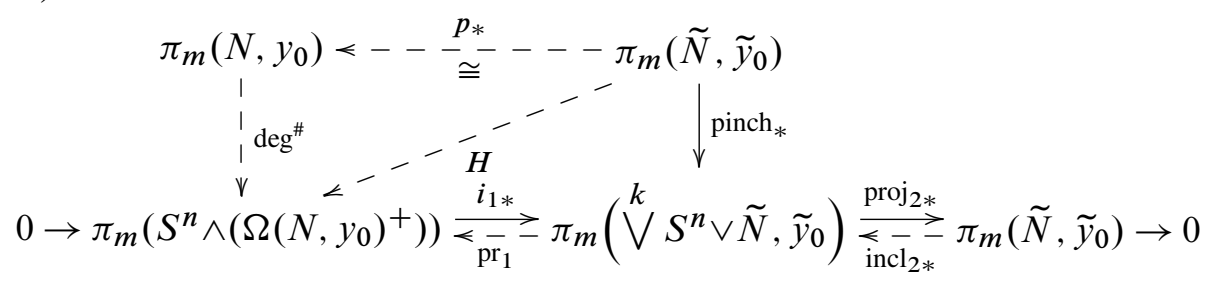

where the horizontal short exact homotopy sequence (of $\operatorname{proj}_{2}$, turned into a fiber map) splits canonically via the inclusion incl $2: \tilde{N} \subset \bigvee S^{n} \vee \tilde{N}$. As we will see below (cf (7-8)) the composite $H$ of the resulting projection $\mathrm{pr}_{1}$ with pinch $_{*}$ is an enriched Hopf-Ganea invariant homomorphism (relative to the attaching map att in (7-1)). But let us first compare it to $\operatorname{deg}^{\#}=\omega^{\#}(, *)$ (cf Proposition 6.1).

Theorem 7.2 $H:=\operatorname{pr}_{1} \circ \operatorname{pinch}_{*}$ coincides with $\operatorname{deg}^{\#} \circ p_{*}$ up to an involution of the target group $\pi_{m}\left(S^{n} \wedge \Omega\left(N, y_{0}\right)^{+}\right)$.

Proof Given $\tilde{f}:\left(I^{m}, \partial I^{m}\right) \longrightarrow\left(\tilde{N}, \tilde{y}_{0}\right)$, we define

$$
u^{\prime}, u^{\prime \prime}, u:\left(I^{m}, \partial I^{m}\right) \longrightarrow\left(\bigvee^{k} S^{n} \vee \tilde{N}, \tilde{y}_{0}\right)
$$

by $u^{\prime}:=\operatorname{pinch} \circ \tilde{f}, u^{\prime \prime}=\operatorname{incl}_{2} \circ \operatorname{proj}_{2} \circ u^{\prime}$ and $u=u^{\prime}-u^{\prime \prime}+\tilde{y}_{0}$ where the three summands have their parameters in $[(i-1) / 3, i / 3] \times I^{m-1}, i=1,2,3$. We lift $u$ to a map $\widehat{u}=\left(u, \widehat{u}_{2}\right)$ into the fiber $F$ of $\operatorname{proj}_{2}(\operatorname{cf}(7-3))$ by putting

$$
\widehat{u}_{2}\left(x_{1}, x^{\prime}\right)=\operatorname{proj}_{2} \circ u \circ\left(\text { straight path from }\left(x_{1}, x^{\prime}\right) \text { to }\left(0, x^{\prime}\right) \text { in } I^{m}\right)
$$

whenever $\left(x_{1}, x^{\prime}\right) \in\left[0, \frac{2}{3}\right] \times I^{m-1}$ and by using the strip $\left[\frac{2}{3}, 1\right] \times I^{m-1}$ for the obvious deformation to make sure that $\widehat{u}$ is constant on $\{1\} \times I^{m-1}$ (and hence on the whole boundary $\partial I^{m}$ of $\left.I^{m}\right)$. Then quot $\circ \widehat{u}$ (cf Lemma 7.1) represents $H([\widetilde{f}]):=$ $p r_{1} \circ \operatorname{pinch}_{*}([\tilde{f}])(\operatorname{cf}(7-4))$. 
We compare this homotopy class to the degree $\operatorname{deg}^{\#}(f)=\omega^{\#}(f, *)$ of $f=p \circ \tilde{f}$ by inspecting the corresponding geometric (Pontryagin-Thom) data (as described eg in (2-8) and (2-10)). We may assume that $* \in N$ is a regular value of $f$. Applying the Pontryagin-Thom procedure to quot $\circ \widehat{u}$ we obtain in the first place the submanifold

$$
u^{-1}\left(\left\{\widetilde{*}_{1}, \ldots, \widetilde{*}_{k}\right\}\right) \subset \stackrel{\circ}{I}^{m} \approx S^{m}-\left\{x_{0}\right\}
$$

which is the inverse image of the zero section $\Omega\left(N, y_{0}\right) \times\{0\}$ in the Thom space $S^{n} \wedge\left(\Omega\left(N, y_{o}\right)^{+}\right)$of the trivial $n$-plane bundle over $\Omega\left(N, y_{0}\right)$ (cf the discussion preceding Lemma 7.1). Up to the dilation $\left(0, \frac{1}{3}\right) \times \stackrel{\circ}{I}^{m-1} \approx \stackrel{\circ}{I}^{m}$ this submanifold is equal to

$$
u^{\prime-1}\left(\left\{\widetilde{*}_{1}, \ldots, \widetilde{*}_{k}\right\}\right)=\tilde{f}^{-1}\left(\left\{\tilde{*}_{1}, \ldots, \widetilde{*}_{k}\right\}\right)=f^{-1}(\{*\})=C(f, *),
$$

ie to the first component of the triple $\left(C(f, *), \widetilde{g}_{1}, \bar{g}^{\#}\right)$ which represents deg ${ }^{\#}(f)$. Also the maps into $\Omega\left(N, y_{0}\right)$ are homotopic (up to reversing the direction of the loops). Indeed, the straight paths which occur in the definition of $\widehat{u}_{2}$ and end in $\{0\} \times I^{m-1}$ correspond to a homotopy $G$ which shifts $C(f, *)$ towards $x_{0}$ as in the construction of $\tilde{g}_{1}$ (cf (2-8)); note that the identity map on $\tilde{N}$ can be deformed into the composite

$$
\tilde{N} \longrightarrow \tilde{N} / \bigvee^{k} B^{n}=\tilde{N}-\bigcup^{k} \stackrel{\circ}{B}^{n} / \bigvee^{k} S^{n-1} \cong \tilde{N}
$$

(the homeomorphisms to the right hand side here and in (7-1) agree). However, the framings of the two Pontryagin-Thom data differ by an automorphism of the trivial $n$-plane bundle induced by $\widetilde{g}_{1}(\mathrm{cf}(2-8)$ and (2-10)).

There is a canonical isomorphism

$$
\kappa: \pi_{m}\left(S^{n} \wedge(\Omega N)^{+}\right) \longrightarrow \pi_{m}\left(\bigvee^{k} S^{n} \vee \tilde{N}, \tilde{N}\right)
$$

since the natural epimorphisms from $\pi_{m}\left(\vee S^{n} \vee \tilde{N}\right)$ onto these groups have the same kernel (cf (7-4)). The isomorphisms $p_{*}$ and $\kappa$ allow a purely homotopy theoretical interpretation of our basic coincidence invariant $\mathrm{deg}^{\#}$ (cf also Remark 7.7).

Corollary 7.3 $\kappa \circ \mathrm{deg}^{\#} \circ p_{*}$ coincides with the composed homomorphism

$$
\pi_{m}(\tilde{N}) \longrightarrow \pi_{m}\left(\tilde{N}, \tilde{N}-\bigcup^{k} \stackrel{\circ}{B^{n}}\right) \stackrel{\text { pinch }_{*}}{\longrightarrow} \pi_{m}\left(\bigvee S^{n} \vee \tilde{N}, \tilde{N}\right)
$$

up to an involution of the target group. 
In particular, the group $X_{m}(N)$ (cf Definition 6.4) is canonically isomorphic to the kernel of

$$
\left(\operatorname{pinch}_{*}, \partial\right): \pi_{m}\left(\tilde{N}, \tilde{N}-\bigcup^{k} \stackrel{\circ}{B}^{n}\right) \longrightarrow \pi_{m}\left(\bigvee^{k} S^{n} \vee \tilde{N}, \tilde{N}\right) \oplus \pi_{m-1}\left(\tilde{N}-\bigcup^{k} \stackrel{\circ}{B}^{n}\right)
$$

where $\partial$ denotes the obvious connecting homomorphism.

Next observe that both homomorphisms $i_{1 *}$ and $\mathrm{pr}_{1}$ in diagram (7-4) commute with $\mathrm{in}_{*}$, ret $_{*}$ (cf (6-7)) on one side and with the homomorphisms induced by the obvious maps

$$
\bigvee S^{n} \underset{\text { proj }_{1}}{\stackrel{\text { incl }_{1}}{\gtrless}} \vee S^{n} \vee \tilde{N}
$$

on the other side. Thus we obtain a canonical decomposition

$$
\pi_{m}\left(S^{n} \wedge\left(\Omega\left(N, y_{0}\right)^{+}\right)\right) \cong \pi_{m}\left(\bigvee^{k} S^{n}\right) \oplus \pi_{m}\left(\left(\bigvee^{k} S^{n}\right) b \tilde{N}\right)
$$

where $\left(\bigvee^{k} S^{n}\right) b \tilde{N}$ denotes the homotopy fiber of the inclusion

$$
\bigvee^{k} S^{n} \vee \tilde{N} \subset\left(\bigvee^{k} S^{n}\right) \times \tilde{N}
$$

(cf Ganea [10, (9)] or Cornea-Lupton-Oprea-Tanré [7, 6.7]). Given $[\tilde{f}] \in \pi_{m}\left(\tilde{N}, \tilde{y}_{0}\right)$, this yields the decomposition

$$
H([\tilde{f}])=\operatorname{pr}_{1} \circ \operatorname{pinch}_{*}([\tilde{f}])=\left([\operatorname{coll} \circ \tilde{f}], H_{\mathcal{C}}(\tilde{f})\right)
$$

(cf (7-4) and Theorem 7.2) where

$$
\text { coll: } \tilde{N} \longrightarrow \tilde{N} /\left(\tilde{N}-\bigcup^{k} \stackrel{\circ}{B}^{n}\right) \cong \bigvee^{k} S^{n}
$$

denotes the collapsing map $(7-1)$ and $H_{\mathcal{C}}(\tilde{f})$ is the $\mathcal{C}-$ Hopf-Ganea invariant of $\tilde{f}$ based on the cofibration

$$
\mathcal{C}: \bigvee_{i=1}^{k} S^{n-1} \subset \tilde{N}-\bigcup_{i=1}^{k} \stackrel{\circ}{B}_{i} \longrightarrow \tilde{N}
$$

(cf Cornea-Lupton-Oprea-Tanré [7, 6.7] or Fernández-Suárez-Gómez-Tato-Tanré [9, $1.1])$. 
Corollary 7.4 Given maps $f, f_{1}, f_{2}:\left(S^{m}, x_{0}\right) \rightarrow\left(N, y_{0}\right)$, let $\tilde{f}, \tilde{f}_{1}, \tilde{f}_{2}$ be the corresponding (basepoint preserving) liftings to the universal covering space $\tilde{N}$.

If $M C(f, *)<\infty$ then $H_{\mathcal{C}}(\tilde{f})=0$.

If $\operatorname{MC}\left(f_{1}, f_{2}\right)<\infty$ then $H_{\mathcal{C}}\left(\tilde{f}_{1}\right)=-\underline{\operatorname{inv}}\left(H_{\mathcal{C}}\left(\tilde{f}_{2}\right)\right)$ where the involution inv of

$$
\pi_{m}\left(\left(\bigvee S^{n}\right) b \tilde{N}\right) \cong \pi_{m}\left(S^{n} \wedge(\Omega N)^{+}\right) / \operatorname{in}_{*}\left(\pi_{m}\left(\bigvee S^{n}\right)\right)
$$

is induced by the involutions in (2-7) and Theorem 7.2.

Proof The involution occurring in Theorem 7.2 (and described at the end of its proof) preserves the subgroup $\operatorname{in}_{*}\left(\pi_{m}\left(\bigvee S^{n}\right)\right)$ of $\pi_{m}\left(S^{n} \wedge(\Omega N)^{+}\right)(\operatorname{cf}(6-7))$. Thus $H_{\mathcal{C}}(\tilde{f})$ coincides (up to an isomorphism) with the class of $\operatorname{deg}^{\#}(f)$ in the quotient group $\pi_{m}\left(S^{n} \wedge(\Omega N)^{+}\right) / \operatorname{in}_{*}\left(\pi_{m}\left(\bigvee S^{n}\right)\right)$. But clearly this class vanishes if $M C(f, *)<\infty$ (see Theorem 3.1(i) and (6-8)). The second claim follows similarly from Proposition 6.1 .

Remark 7.5 Our interpretation of the Hopf-Ganea invariant $H_{\mathcal{C}}$ as an obstruction can be extended considerably: given any natural number $k$, an embedding of $\bigvee^{k} B^{n}$ into any simply connected $n$-manifold $\tilde{N}$, and any map $\tilde{f}: S^{m} \rightarrow \tilde{N}, H_{\mathcal{C}}(\tilde{f})$ (cf (7-9)) must vanish if $\tilde{f}$ is homotopic to a map with at least $k$ almost injective points (cf Definition 6.12).

In view of the last corollary it is natural to ask whether the Hopf-Ganea invariant is the only finiteness obstruction. In order to get a partial answer consider the commuting diagram (where $m \geq 2$ as before)

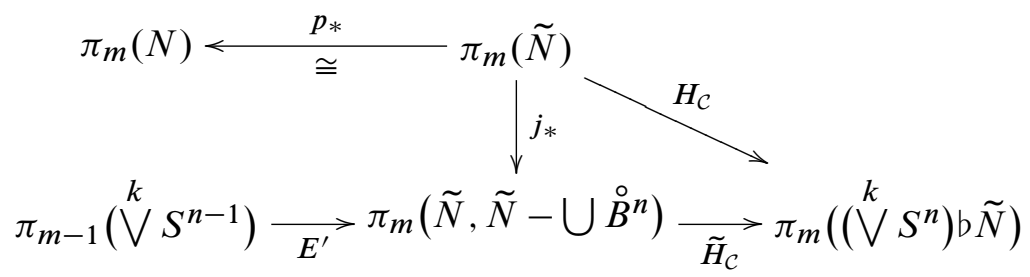

(compare Fernández-Suárez-Gómez-Tato-Tanré [9, Section 1]). Here $E^{\prime}$ denotes the 'suspension homomorphism' described by straight paths in $\left(\bigvee B^{n}, \bigvee S^{n-1}\right) \subset$ $\left(\tilde{N}, \tilde{N}-\cup \stackrel{\circ}{B}^{n}\right)$ and $\widetilde{H}_{\mathcal{C}}$ is a canonical extension of the $\mathcal{C}$-Hopf-Ganea homomorphism (cf (7-9)) to the indicated relative homotopy group. Given $[\tilde{f}] \in \pi_{m}(\tilde{N})$, we see (as in the proof of Theorem 6.9) that $M C(p \circ \widetilde{f}, *)<\infty$ if and only if $j_{*}([\tilde{f}])$ lies in $E^{\prime}\left(\oplus^{k} \pi_{m-1}\left(S^{n-1}\right)\right)$ (provided $m \geq 3$ or $\pi_{1}(N)=0$ ). 
Theorem 7.6 Assume that $N$ is $q$-connected and $2 \leq m \leq q+2 n-3$. Then we have for all $[\tilde{f}] \in \pi_{m}(\tilde{N})$ :

$$
M C(p \circ \tilde{f}, *) \text { is finite if and only if } H_{\mathcal{C}}(\tilde{f})=0 .
$$

Assume in addition that $E: \pi_{m-1}\left(S^{n-1}\right) \longrightarrow \pi_{m}\left(S^{n}\right)$ is injective. Then $X_{m}(N)=0$.

Proof When $n=2<m$ then $N$ must be open here and $M C(p \circ \tilde{f}, *)=0$ (cf Corollary 6.3). Thus we may assume that $n \geq 3$. Since $\tilde{N}$ is at least $(m-2 n+3)$-connected, so is the (first) inclusion in $\mathcal{C}$ ( $\mathrm{cf}(7-9)$; use Whitehead's theorem and excision in homology). Therefore the horizontal line in diagram (7-10) is part of an exact EHP-sequence (cf [9, 1.3]). Moreover $E^{\prime}\left(\oplus^{k} \pi_{m-1}\left(S^{n-1}\right)\right)$ is the full image of $E^{\prime}$ since $k=\# \pi_{1}(N)=1$ or else $m-1<2 n-3$ (compare Hilton [13]). Our claim follows from the previous discussion and from Theorem 1.14.

Remark 7.7 We may also consider the $\mathcal{C}^{\prime}$-Hopf-Ganea homomorphism

$$
H_{\mathcal{C}^{\prime}}: \pi_{m}(N) \longrightarrow \pi_{m}\left(S^{n} \mathrm{~b} N\right)
$$

based on the cofibration

$$
\mathcal{C}^{\prime}: S^{n-1} \subset N-\stackrel{\circ}{B}^{n} \longrightarrow N
$$

where $S^{n-1}$ is the boundary of some embedded $n$-ball $B^{n}$ ("top cell") in $N$ (compare $(7-8)$ and (7-9)). $H_{\mathcal{C}^{\prime}}$ is induced by the corresponding pinch map

$$
\text { pinch' }^{\prime} N \longrightarrow N / S^{n-1} \approx S^{n} \vee N
$$

and the second projection in the canonical decomposition

$$
\pi_{m}\left(S^{n} \vee N\right) \cong \pi_{m}\left(S^{n} \wedge(\Omega N)^{+}\right) \oplus \pi_{m}(N) \cong \pi_{m}\left(S^{n}\right) \oplus \pi_{m}\left(S^{n} \mathrm{~b} N\right) \oplus \pi_{m}(N)
$$

(compare (7-1), (7-4), and (7-7)); here the first component homomorphism of $\operatorname{pinch}_{*}^{\prime}$ is induced by a collapsing map coll' $: N \rightarrow S^{n}$ of degree \pm 1 (as in (2-11)).

According to Theorem 7.2 the combined homomorphism

$$
\left(\operatorname{coll}_{*}^{\prime}, H_{\mathcal{C}^{\prime}}\right): \pi_{m}(N) \longrightarrow \pi_{m}\left(S^{n}\right) \oplus \pi_{m}\left(S^{n} \mathrm{~b} N\right)
$$

agrees with $\mathrm{deg}^{\#}$ up to an isomorphism between the target groups. In particular, for all $[f] \in \pi_{m}(N)$ the Hopf-Ganea invariant $H_{\mathcal{C}^{\prime}}([f])$ (relative to the attaching class of the top cell of $N$ ) can be described entirely by the coincidence data of the preimage $f^{-1}(\{*\})$. To be more precise assume that $f$ is smooth with regular value $* \in N$. Then $\operatorname{coll}_{*}^{\prime}([f])$ corresponds (via Pontryagin-Thom) to the bordism class of the framed submanifold $C:=C(f, *)=f^{-1}(\{*\})$ of $S^{m}-\left\{x_{0}\right\}$ and $H_{\mathcal{C}^{\prime}}([f])$ measures the 
added information which the lifting $\widetilde{g}(\operatorname{cf}(1-5))$ or, equivalently, the map $\widetilde{g}_{1}: C \rightarrow \Omega N$ contributes to $\operatorname{deg}^{\#}([f])=\left[f^{-1}(\{*\}), \widetilde{g}_{1}, \bar{g}_{1}^{\#}\right](\operatorname{cf}(2-8)-(2-10))$. On the other hand, the element $\operatorname{coll}_{*}([\tilde{f}]) \in \pi_{m}\left(\vee^{k} S^{m}\right)(\operatorname{cf}(7-7),(7-8))$ corresponds to the link $C=\amalg C_{\alpha}$ of disjoint framed submanifolds (ie it captures the Nielsen decomposition of $C$ determined by $\tilde{g}$, cf (2-5)), and the Ganea-Hopf invariant $H_{\mathcal{C}}([\tilde{f}])$, which we studied in (7-8)(7-10), Corollary 7.4 and Theorem 7.6, measures the remaining information contained in $\operatorname{deg}^{\#}([f])$.

In general, $H_{\mathcal{C}^{\prime}}([f])$ contains much more information than $H_{\mathcal{C}}([\tilde{f}])$. Indeed,

$$
\pi_{m}\left(S^{n} \mathrm{~b} N\right) \cong \operatorname{ker}\left(\operatorname{ret}_{*}^{\prime}\right) \oplus \pi_{m}\left(\left(\bigvee^{k} S^{n}\right) b \tilde{N}\right)
$$

where ret': $\vee^{k} S^{n} \rightarrow S^{n}$ maps each sphere in the wedge identically to $S^{n}$. In view of the Hilton decomposition

$$
\pi_{m}\left(\bigvee^{k} S^{n}\right) \cong \bigoplus_{k} \pi_{m}\left(S^{n}\right) \oplus \bigoplus_{\substack{k \\ 2}} \pi_{m}\left(S^{2 n-1}\right) \oplus \ldots
$$

(cf Hilton [13]) the kernel of ret $_{*}^{\prime}$ in (7-16) may be highly nontrivial whenever $k>1$.

In particular, $H_{\mathcal{C}^{\prime}}([f])$ need not vanish when $M C(f, *)$ is finite (eg in the case $m=n$ and $\# G>1$ in Example 1.13). Thus it is suitable to use the universal covering space $\tilde{N}$ and the corresponding Hopf-Ganea homomorphism $H_{\mathcal{C}}([\tilde{f}])$ when we develop such finiteness criteria as Corollary 7.4 and Theorem 7.6. However, $\tilde{N}$ is not required in Corollary 7.3: clearly $X_{m}(N)$ is also canonically isomorphic to the kernel of the obvious homomorphism

$$
\left(\operatorname{pinch}_{*}^{\prime}, \partial^{\prime}\right): \pi_{m}\left(N, N-\stackrel{\circ}{B}^{n}\right) \longrightarrow \pi_{m}\left(S^{n} \vee N, N\right) \oplus \pi_{m-1}\left(N-\stackrel{\circ}{B}^{n}\right) .
$$

\section{Appendix A Base points}

It is sometimes useful to require that the maps $f_{1}$ and $f_{2}$ as well as their homotopies preserve base points. As we will see this has no impact on our numerical invariants whenever $m, n \geq 1$.

Let $x_{0} \in M$ and $y_{1} \neq y_{2} \in N$ be a given choice of base points. For any two "base point preserving" maps

$$
f_{i}:\left(M, x_{0}\right) \longrightarrow\left(N, y_{i}\right), \quad i=1,2,
$$


define $M C C_{b}\left(f_{1}, f_{2}\right)$ (and $M C_{b}\left(f_{1}, f_{2}\right)$, resp.) to be the minimum number of path components (and of points, resp.) in the coincidence locus $C\left(f_{1}^{\prime}, f_{2}^{\prime}\right)$ of any pair of maps in the same base point preserving homotopy classes, ie

$$
\left[f_{i}^{\prime}\right]=\left[f_{i}\right] \in\left[\left(M, x_{0}\right),\left(N, y_{i}\right)\right], \quad i=1,2 .
$$

Lemma A $\operatorname{MCC}_{b}\left(f_{1}, f_{2}\right)=\operatorname{MCC}\left(f_{1}, f_{2}\right)$ and $M C_{b}\left(f_{1}, f_{2}\right)=M C\left(f_{1}, f_{2}\right)$; in particular if the pair $\left(f_{1}, f_{2}\right)$ is loose then there are already base point preserving homotopies which deform $f_{1}$ and $f_{2}$ away from one another.

(For a related result in the context of classical fixed point theory see eg Jiang [15, Section 3]).

Proof The claims concerning $M C C$ and $M C$ will be treated simultaneously. We may concentrate on the case $m, n \geq 2$.

Clearly $M(C) C_{b}\left(f_{1}, f_{2}\right) \geq M(C) C\left(f_{1}, f_{2}\right)$.

Conversely, let the minimum number $M(C) C\left(f_{1}, f_{2}\right)$ be realized by a pair $\left(f_{1}^{\prime \prime}, f_{2}^{\prime \prime}\right)$. Without changing the number of coincidence components (or points, resp.) we may - in a first step - deform this pair until it preserves base points. Indeed, if there is a point $\widehat{x}_{0} \in M$ such that $f_{1}^{\prime \prime}\left(\widehat{x}_{0}\right) \neq f_{2}^{\prime \prime}\left(\widehat{x}_{0}\right)$, compose $\left(f_{1}^{\prime \prime}, f_{2}^{\prime \prime}\right)$ with isotopies in $M$ and $N$ which move $x_{0}$ to $\widehat{x}_{0}$ and $f_{i}\left(\widehat{x}_{0}\right)$ to $y_{i}, i=1,2$; if $f_{1}^{\prime \prime} \equiv f_{2}^{\prime \prime}$ remove first a small ball $\stackrel{\circ}{B} \subset M$ from the coincidence set $C\left(f_{1}^{\prime \prime}, f_{2}^{\prime \prime}\right)$ by "pushing $f_{2}^{\prime \prime}$ slightly off $f_{1}^{\prime \prime}$ in $\stackrel{\circ}{B} "$.

The second step is a simple modification near $x_{0}$ which makes $f_{i}^{\prime \prime}$ homotopic to $f_{i}$ in the base point preserving sense, $i=1,2$. Let

$$
c_{i}=H_{i}\left(x_{0},-\right):(I,\{0,1\}) \longrightarrow\left(N, y_{i}\right)
$$

be smooth transverse paths resulting from homotopies $H_{i}: f_{i} \sim f_{i}^{\prime \prime}, i=1,2$. After suitable changes of the parametrization near finitely many intersection points $c_{1}$ and $c_{2}$ will be coincidence free. Now identify a small ball in $M$ around $x_{0}$ with the unit ball $B^{m}$ in $\mathbb{R}^{m}$. We may assume that for every $x \in B^{m}, f_{i}^{\prime \prime}(x)=y_{i}$; then replace this by $f_{i}^{\prime \prime}(x):=c_{i}(\|x\|), i=1,2$. This procedure does not change the coincidence set of $\left(f_{1}^{\prime \prime}, f_{2}^{\prime \prime}\right)$ but yields basepoint preserving homotopies. Thus in the end we conclude that $M(C) C_{b}\left(f_{1}, f_{2}\right) \leq \#\left(\pi_{0}\right) C\left(f_{1}^{\prime \prime}, f_{2}^{\prime \prime}\right)=M(C) C\left(f_{1}, f_{2}\right)$. 


\section{References}

[1] MF Atiyah, R Bott, A Shapiro, Clifford modules, Topology 3 (1964) 3-38 MR0167985

[2] S A Bogaty̆, D L Gonçalves, $\mathbf{Z} \mathbf{H}$, Coincidence theory: the minimization problem, $\mathrm{Tr}$. Mat. Inst. Steklova 225 (1999) 52-86 MR1725933 English translation: Proc. Steklov Inst. Math. 225 (1999) 45-77

[3] R B S Brooks, On removing coincidences of two maps when only one, rather than both, of them may be deformed by a homotopy, Pacific J. Math. 40 (1972) 45-52 MR0341470

[4] R F Brown, Wecken properties for manifolds, from: "Nielsen theory and dynamical systems (South Hadley, MA, 1992)", Contemp. Math. 152, Amer. Math. Soc., Providence, RI (1993) 9-21 MR1243467

[5] R F Brown, H Schirmer, Nielsen coincidence theory and coincidence-producing maps for manifolds with boundary, Topology Appl. 46 (1992) 65-79 MR1177164

[6] O Cornea, New obstructions to the thickening of $\mathrm{CW}$-complexes, Proc. Amer. Math. Soc. 132 (2004) 2769-2781 MR2054804

[7] O Cornea, G Lupton, J Oprea, D Tanré, Lusternik-Schnirelmann category, Mathematical Surveys and Monographs 103, American Mathematical Society, Providence, RI (2003) MR1990857

[8] A Dold, D L Gonçalves, Self-coincidence of fibre maps, Osaka J. Math. 42 (2005) 291-307 MR2147735

[9] L Fernández-Suárez, A Gómez-Tato, D Tanré, Hopf-Ganea invariants and weak LS category, Topology Appl. 115 (2001) 305-316 MR1848131

[10] T Ganea, A generalization of the homology and homotopy suspension, Comment. Math. Helv. 39 (1965) 295-322 MR0179791

[11] M J Greenberg, J R Harper, Algebraic topology, Mathematics Lecture Note Series 58, Benjamin/Cummings Publishing Co. Advanced Book Program, Reading, Mass. (1981) MR643101

[12] A Hatcher, F Quinn, Bordism invariants of intersections of submanifolds, Trans. Amer. Math. Soc. 200 (1974) 327-344 MR0353322

[13] P J Hilton, On the homotopy groups of the union of spheres, J. London Math. Soc. 30 (1955) 154-172 MR0068218

[14] J Jezierski, The least number of coincidence points on surfaces, J. Austral. Math. Soc. Ser. A 58 (1995) 27-38 MR1313883

[15] B Jiang, Fixed points and braids, Invent. Math. 75 (1984) 69-74 MR728139

[16] B J Jiang, Fixed points and braids. II, Math. Ann. 272 (1985) 249-256 MR796251 
[17] U Koschorke, Vector fields and other vector bundle morphisms-a singularity approach, Lecture Notes in Mathematics 847, Springer, Berlin (1981) MR611333

[18] U Koschorke, Coincidence theory in arbitrary codimensions: the minimizing problem, Oberwolfach Reports (2004)

[19] U Koschorke, Linking and coincidence invariants, Fund. Math. 184 (2004) 187-203 MR2128050

[20] U Koschorke, Selfcoincidences in higher codimensions, J. Reine Angew. Math. 576 (2004) 1-10 MR2099198

[21] U Koschorke, Geometric and homotopy theoretic methods in Nielsen coincidence theory, Fixed Point Theory and Appl. to appear (2006)

[22] U Koschorke, Nielsen coincidence theory in arbitrary codimensions, J. Reine Angew. Math., to appear (2006) arXiv:math.AT/0408044

[23] U Koschorke, B Sanderson, Geometric interpretations of the generalized Hopf invariant, Math. Scand. 41 (1977) 199-217 MR0474289

[24] G W Whitehead, Elements of homotopy theory, Graduate Texts in Mathematics 61, Springer, New York (1978) MR516508

[25] A Wyler, Sur certaines singularités d'applications de variétés topologiques, Comment. Math. Helv. 42 (1967) 28-48 MR0214075

Universität Siegen, Emmy Noether Campus, Walter-Flex-Str. 3

D-57068 Siegen, Germany

koschorke@mathematik.uni-siegen.de

http: //www . math . uni-siegen.de/topology/

Proposed: Shigeyuki Morita

Seconded: Wolfgang Lück, Tom Goodwillie

Received: 29 September 2005

Revised: 9 March 2006 